\title{
A DEGENERATION OF THE MODULI SPACE OF STABLE BUNDLES
}

\author{
D. GIESEKER
}

1. Let $k$ be an algebraically closed field and let $d$ be an odd integer. Let $g \geqslant 2$ be an integer and suppose $Y$ is a smooth projective curve of genus $g$. Let $U_{Y}$ be the set of isomorphism classes of stable bundles $E$ of rank two and degree $d$. (Note: we do not fix $\wedge^{2} E$.) Following Mumford and Seshadri, we know that $U_{Y}$ is in a natural way the set of (closed) points of a smooth projective variety again denoted by $U_{Y}$. Our aim in this paper is to develop a method of studying the topology of $U_{Y}$ by degeneration methods. Our main application is the proof of the following theorem conjectured by Newstead and Ramanan.

Theorem $1.1(k=\mathrm{C})$. The kth Chern class of the tangent bundle of $U_{Y}$ is zero in the deRham cohomology of $U_{Y}$ if $k>2 g-2$.

We hope that degeneration methods may be useful in other contexts. For instance, one can hope that the theory can be generalized to bundles of arbitrary degree and rank. One should also be able to compute the lower Chern classes of $\Omega_{U_{Y}}^{1}$.

The following is a brief outline of this paper: Let $X_{0}$ be an irreducible curve of genus $g$ which is smooth except of one ordinary node $N$. We let $X$ be the normalization of $X_{0}$ and let $P_{1}$ and $P_{2}$ be the inverse image of $N$. Our object is to find a (singular) projective variety $U_{X_{0}}$ which will play the role to $U_{Y}$ when $Y$ is smooth. In particular, if $\left\{Y_{t}\right\}$ is a family of smooth curves degenerating to $X_{0}$, then we desire that $U_{Y_{t}}$ generates to $U_{X_{0}}$.

The first difficulty in constructing $U_{X_{0}}$ is that one cannot hope that all the points of $U_{X_{0}}$ will correspond to actual bundles on $X_{0}$. There are two methods to resolve this difficulty. One is to consider certain torsion-free sheaves on $X_{0}$ to obtain a candidate for $U_{X_{0}}$ [3]. However, such a $U_{X_{0}}$ does not appear to have (analytic) normal crossings. The second method, which we will follow, is to consider certain bundles on certain semistable models of $X_{0}$ as is suggested by

Received January 10, 1983 and, in revised form, July 7, 1983. This work was partially supported by National Science Foundation Grant MCS77-18723 A04. 
the theory developed in [1]. The construction of $U_{X_{0}}$ and the examination of its local properties occupy $\S \S 3$ and 4 . ( $\$ 2$ consists of definitions and elementary results on stable bundles on smooth curves.)

Let $U$ be the set of all bundles $E$ on $X_{0}$ so that the pullback $\tilde{E}$ of $E$ to $X$ is stable. We will see $U$ is a smooth open subvariety of $U_{X_{0}}$. Letting $\tilde{U}_{X_{0}}$ be the normalization of $U_{X_{0}}$, we see $\tilde{U}_{X_{0}}$ is a smooth compactification of $U$. On the other hand, $U$ is a fiber bundle with fiber GL(2) over $S_{0}$, the moduli space of stable bundles on $X$. The main object of the paper is to embed $U$ into a certain projective fiber bundle $S_{3}$ over $U_{0}$ and then to obtain $\tilde{U}_{X_{0}}$ by blowing up and then blowing down $S_{3}$ in a fairly explicit way. The main result is Theorem 13.1. $\S \S 11$ and 12 are independent of the rest of the paper. $\$ 11$ gives conditions which insure a birational map between projective varieties is obtained by blowing up the target. $\$ 12$ consists of a Chern class computation which together with Theorem 13.1 proves Theorem 1.1.

Since $d$ is odd, we let $d=2 \alpha+1$.

I wish to thank Jim Carlson, Herb Clemens and Steve Zucker for their help on mixed Hodge structures.

2. We begin with some terminology. Let $S$ be a scheme of finite type over $k$ and let $Z$ be a closed subscheme. Let $E$ be a bundle on $S$.

Definition 1.1. A $Z$ quotient of $E$ is a locally free sheaf of $\theta_{Z}$ modules $Q$ and a surjection $\varphi: E \rightarrow Q$.

Two $Z$ quotients $E \rightarrow Q$ and $E \rightarrow Q^{\prime}$ are equal if $\operatorname{Ker}(E, Q)=\operatorname{Ker}\left(E, Q^{\prime}\right)$. We call $E^{\prime}=\operatorname{Ker}(E, Q)$ the modification of $E$ at $Q$. Let $F=\operatorname{Ker}\left(E_{Z}, Q\right)$. Then $F$ is a sheaf of locally free $\theta_{Z}$ modules and $F$ is a $Z$ quotient of $E^{\prime}$. We call $F$ the canonical $Z$ quotient of the modification of $E$ at $Q$. If $g_{Z}$, the ideal sheaf of $Z$ in $S$, is invertible, then $E^{\prime}$ is a bundle.

If $Z^{\prime} \subseteq Z$, we call $Q \otimes \vartheta_{Z^{\prime}}$ the $Z^{\prime}$ quotient induced from $Q$. If $Z^{\prime \prime}$ is another closed subscheme of $S$, and $Q^{\prime \prime}$ is a $Z^{\prime \prime}$ quotient of $E$ and $W \subseteq Z \cap Z^{\prime \prime}$, then we say $Q$ and $Q^{\prime \prime}$ coincide over $W$ if the induced $W$ quotients are equal.

Remark 2.2. Suppose $S$ is smooth, $Z_{1}$ and $Z_{2}$ are two divisors intersecting transversally, and $Q$ is a $Z_{1}$ quotient of $E$. Let $Q^{\prime}$ be the induced $Z_{1} \cap Z_{2}$ quotient of $E_{Z_{2}}, E^{\prime}=\operatorname{Ker}(E, Q)$ and $E^{\prime \prime}=\operatorname{Ker}\left(E_{Z_{2}}, Q^{\prime}\right)$. Then $\left(E^{\prime}\right)_{Z_{2}}=E^{\prime \prime}$, and the canonical quotients coincide over $Z_{1} \cap Z_{2}$.

Now let $C$ be a smooth projective curve, $E$ a bundle of rank $r$ over $C$ and $Q$ a locally free quotient of $E$.

Definition 2.3. $Q$ is destabilizing (resp. semistabilizing) if

$$
\frac{\operatorname{deg} Q}{\operatorname{rk} Q}<\frac{\operatorname{deg} E}{\operatorname{rk} E} \quad\left(\text { resp. } \frac{\operatorname{deg} Q}{\operatorname{rk} Q}=\frac{\operatorname{deg} E}{\operatorname{rk} E}\right) .
$$


Now if $C$ is rational, then $E$ is a direct sum of line bundles $L_{i}$.

Definition 2.4. $E$ is standard on a rational curve if $\operatorname{deg} L_{i}$ is either zero or one for each $i$.

If $E$ is standard, there is a unique quotient $Q$ so that $Q$ is a direct sum of $\vartheta_{C}$ 's and $\operatorname{Ker}(E, Q)$ is a direct sum of $\theta(1)$ 's. We call $Q$ the standard quotient of $E$.

$X$ will be a fixed nonsingular curve of genus $g-1$ for the rest of the paper and $P_{1}$ and $P_{2}$ will be fixed distinct points on $X$. Let $E$ be a bundle of rank two on $X$ and let $L_{i}$ be $P_{i}$ quotients of $E$ of rank one. We suppose $E$ is stable of odd degree $d=2 \alpha+1$.

Lemma 2.5. (i) $E^{\prime}=\operatorname{Ker}\left(E \rightarrow L_{1}\right)$ is semistable. Further there is no semistabilizing quotient $M$ of $E^{\prime}$ which coincides with the canonical $P_{1}$ quotient of $E^{\prime}$.

(ii) $E^{\prime \prime}=\operatorname{Ker}\left(E \rightarrow L_{1} \oplus L_{2}\right)$ is stable unless there is an invertible quotient $L$ of $E$ of degree $\alpha+1$ which coincides with $L_{1}$ and $L_{2}$.

Proof. Consider case (i). Let $M$ be a quotient of $E^{\prime}$ of degree $e \leqslant \alpha$ and let $M^{\prime}=\operatorname{Ker}\left(E^{\prime}, M\right)$. If $e<\alpha$, then $\operatorname{deg} M^{\prime}>\alpha$ so the subline bundle of $E$ containing $M^{\prime}$ is destabilizing. If $e=\alpha$ and $M$ coincides with the canonical quotient of $E^{\prime}$ at $P_{1}$, then the map from $M^{\prime}$ to $E$ vanishes at $P_{1}$ and so $M^{\prime}(P)$ maps to $E$. Then $M^{\prime}(P)$ is destabilizing for $E$.

Consider case (ii). Then $E^{\prime \prime}$ has a quotient $M$ of degree $e \leqslant \alpha-1$ and hence a subbundle $M^{\prime}$ of degree $\geqslant \alpha$. But $M^{\prime}$ must be a subbundle of degree $\alpha$ of $E$, since otherwise $E$ would have a subbundle of degree $>\alpha$. On the other hand, $M^{\prime}$ maps to zero in $L_{1}$ and $L_{2}$, so $E / M^{\prime}$ is a quotient of degree $\alpha+1$ which coincides with $L_{P_{1}}$ and $L_{P_{2}}$.

Lemma 2.6. Suppose $\operatorname{deg} E=2 \alpha+1$ and that $E$ has a quotient $Q$ of degree $\alpha$. Further, suppose the $L_{i}$ do not coincide with $Q$ over $P_{i}$. Then:

(i) $\operatorname{Ker}\left(E, L_{1}\right)=E^{\prime}$ is semistable. Further, $Q$ is a quotient of $E^{\prime}$ and the canonical $P_{1}$ quotient of $E^{\prime}$ is glued to $Q$ over $P_{1}$.

(ii) $\operatorname{Ker}\left(E, L_{1} \oplus L_{2}\right)$ is stable unless there is a quotient $Q^{\prime}$ of $E$ of degree $\alpha+1$ which coincides with $L_{i}$ over $P_{i}$.

The proof of Lemma 2.6 is similar to that of Lemma 2.5 .

3. Recall that $X$ is a fixed nonsingular curve of genus $g-1$. We let $X_{0}$ be the stable curve obtained by identifying $P_{1}$ with $P_{2}$. Now for each $n \geqslant 1$, we can define a semistable curve $X_{n}$ whose components are $X$ and nonsingular rational curves $R_{1}, \cdots, R_{n}$ with $R_{i}$ meeting $R_{i-1}$ and $R_{i+1}$ and $R_{1}$ meeting $P_{1}$ and $R_{n}$ meeting $P_{2}$. 


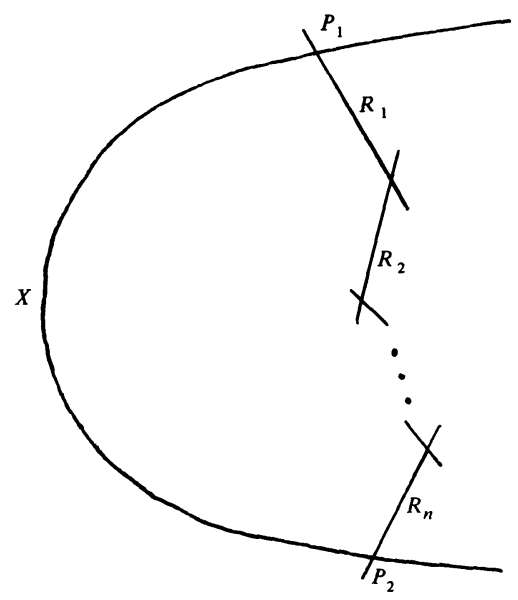

Now we say a bundle $\tilde{E}$ of degree $2 \alpha+1$ on $X$ is slightly unstable if it has a destabilizing quotient of degree $\alpha$.

Now if $E$ is a bundle of rank 2 on $X_{0}$, we let $\tilde{E}$ denote the pullback of $E$ to $X$. If $E$ is a bundle on $X_{i}$, we let $\tilde{E}=E \otimes \vartheta_{X}$. We say $E$ on $X_{i}$ is weakly stable if it has no quotient line bundles $Q$ which are destabilizing, i.e.,

$$
2 \operatorname{deg}_{X_{i}} Q \leqslant \operatorname{deg}_{X_{i}} E \text {. }
$$

We now consider certain types of bundles defined on $X_{0}, X_{1}$ and $X_{2}$. We will assume all types are weakly stable of degree $2 \alpha+1$.

Type $\mathrm{I}_{s}: E$ defined on $X_{0}$ and $\tilde{E}$ is stable.

Type $\mathrm{I}_{u}: E$ defined on $X_{0}$ and $\tilde{E}$ is slightly unstable.

Type $\mathrm{II}_{1}: E$ defined on $X_{1}, \tilde{E}$ is semistable, and $E_{R_{1}}$ is standard of degree 1, i.e., $E_{R_{1}} \cong \vartheta(1) \oplus \Theta$.

Type $\mathrm{II}_{2}: E$ is defined on $X_{1}, \tilde{E}$ is stable and $E_{R_{1}}$ is standard of degree 2, i.e., $E_{R_{1}} \cong \theta(1) \oplus \theta(1)$.

Type III: $E$ is defined on $X_{2}, \tilde{E}$ is stable, $E_{R_{i}}$ is standard of degree 1 , and the standard quotients of $E_{R_{1}}$ and $E_{R_{2}}$ do not coincide over $R_{1} \cap R_{2}$.

Bundles of any of the above types will be called potentially stable. Now recall the setup of [1]. We let $d=2 \alpha+1$ and $n=d+2-2 g$. We let $W$ be a vector space of dimension $n, G$ be the grassmannian of all codimension two subspaces of $W$ and $\varepsilon$ be the universal bundle on $G$. We let $S_{g, d}$ be the Hilbert scheme of curves of degree $d$ and genus $g$ on $G$. We can then consider the stability of the $m$-Hilbert point of $C \in S_{g, d}$ for $m \gg 0$. Our first main result was that there was a $d$ and an $m$ so that if $C$ is smooth, then $C$ is $m$-Hilbert stable if and only if $\varepsilon_{C}$ is stable. Our second main result was that if $C$ is $m$-Hilbert semistable, then $C$ is semistable as a curve and $H^{1}(C, \mathcal{E})=0$. Further, if $C$ is isomorphic to $X_{n}$, then $\mathscr{E}_{C}$ is potentially stable. We fix such a $d$ and $m$. 
Proposition 3.1. Any potentially stable bundle on $X_{n}$ is $m$-Hilbert semistable.

Proof. Let $U$ be the set of $m$-Hilbert stable points. First notice that if $\varepsilon_{C}$ is potentially stable, then $C \in \bar{U}$. Indeed, we can deform any semistable curve to a smooth curve, and we can then lift our bundle to this deformation. On the other hand, on a smooth curve any bundle can be deformed to a stable bundle. Indeed, we can write our bundle and a stable bundle as an extension of the same two line bundles. Since stability is an open condition, our claim follows.

Consequently, if $C \cong X_{n}$, we can find a smooth curve $S$, a family of semistable curves $\pi: Y \rightarrow S$, and a bundle $F$ on $Y$ so that for some $R \in S, F_{R}$ on $Y_{R}=\pi^{-1}(R)$ is our potentially stable bundle and for $Q \neq R, Y_{Q}$ is smooth and $F_{Q}$ is stable. After replacing $S$ by a cover possible ramified over $R$, we can assume there is a family $\pi^{\prime}: Y^{\prime} \rightarrow S$ and a bundle $F^{\prime}$ on $Y^{\prime}$ so that $\left(Y^{\prime}, F^{\prime}\right)$ is isomorphic to $(Y, F)$ over $S-R$ and so that $F_{R}^{\prime}$ is $m$-Hilbert semistable. Now let $Y^{\prime \prime}$ be the smooth surface which is the minimum model obtained by a resolution of singularities of $Y$. By uniqueness of minimum models, $Y^{\prime \prime}$ is also a minimum model of $Y^{\prime}$. We will denote the pullbacks of $F$ and $F^{\prime}$ to $Y^{\prime \prime}$ by $F$ and $F^{\prime}$ again. We have an isomorphism $\psi$ of $F$ with $F^{\prime}$ over $S-R$. Now locally around $R$, we can choose a uniformizing parameter $t$ of $S$ at $R$ and we can find a map $\varphi: F \rightarrow F^{\prime}$ which is not identically zero on $Y_{R}^{\prime \prime}$. Indeed, we just take $\varphi=t^{k} \psi$ for some suitable $k$. Now let $E$ and $E^{\prime}$ be $F_{R}$ and $F_{R}^{\prime}$ on $Y_{R}^{\prime \prime}=Y_{0} . Y_{0}$ is isomorphic to $X_{n}$ for some $n$. We let $R$ be the union of the $R_{i}$ 's. We have nonzero maps $\varphi$ and $\varphi^{\prime}$ from $E$ to $E^{\prime}$ and from $E^{\prime}$ to $E$, and both $E$ and $E^{\prime}$ are the pullbacks of potentially stable bundles. Our main claim is that if $\operatorname{deg} \tilde{E} \geqslant$ $\operatorname{deg} \tilde{E}^{\prime}$, then $\varphi$ is an isomorphism. Our proof of this claim will only use the fact that $E$ and $E^{\prime}$ are the pullbacks of potentially stable bundles, so Proposition 3.1 follows by reversing the roles of $E$ and $E^{\prime}$ if necessary.

We first claim that if $\varphi$ vanishes at $P_{1}$ and $P_{2}$, then $\varphi$ vanishes on $R$. Indeed, we can write $E_{R}=L_{1} \oplus L_{2}$ and $E_{R}^{\prime}=M_{1} \oplus M_{2}$ where each $L_{i}$ and $M_{i}$ has degree zero on all components of $R$ except perhaps for one on which it has degree one. Now

$$
\operatorname{Hom}_{\vartheta_{R}}\left(E_{R}, E_{R^{\prime}}^{\prime}\right)=\bigoplus_{i, j}\left(L_{i}^{-1} \otimes M_{j}\right)
$$

Our assumption means that the components $\varphi_{i j}$ of $\varphi$ vanish at $P_{1}$ and $P_{2}$. But $L_{i}^{-1} \otimes M_{j}$ has nonpositive degree on all components except perhaps for one $R_{k}$. Thus $\varphi_{i j}$ vanish on all components except $R_{k}$, and hence vanish on $R_{k}$, since $\varphi_{i j}$ vanishes at two points of $R_{k}$.

Second, we claim that if $\varphi$ is an isomorphism at $P_{1}$ and $P_{2}$ and $\operatorname{deg} E_{R}=$ $\operatorname{deg} E_{R}^{\prime}$, then $\varphi$ is an isomorphism over $R$. Consider $L=\left(\wedge^{2} E_{R}\right)^{-1} \otimes \wedge^{2} E_{R}^{\prime}$. $L$ has degree zero. First consider the case $\operatorname{deg} E_{R}^{\prime} \leqslant 1$. Then $L$ is either trivial on all components, or has degree one on some component $R_{i}$ and degree -1 on 
$R_{j}$. If $L$ is trivial, then $\wedge^{2} \varphi$ is nowhere zero and so $\varphi$ is an isomorphism. Otherwise, $\wedge^{2} \varphi$ vanishes on $R_{j}$ and hence on all components above or below $R_{j}$ depending on whether $R_{i}$ is below or above $R_{j}$. So $\wedge^{2} \varphi$ is zero at $P_{1}$ or $P_{2}$.

Next consider the case $\operatorname{deg} E_{R}^{\prime}=2$. The arguments given above eliminate all cases except if $L$ has degree one on $R_{k}$ and $R_{l}$ and has degree zero on $R_{m}$ if $m<k$ or $m>l$. In this case, let $R^{\prime}$ be the chain $R_{k+1} \cup \cdots \cup R_{l-1}$. Then $E_{R^{\prime}}^{-1}$ has no sections since it is a direct sum $\left(L_{1}^{-1} \oplus L_{2}^{-1}\right)_{R}$ where $L_{1}$ and $L_{2}$ both have degree one on some component of $R^{\prime}$. Since $E_{R^{\prime}}^{\prime}$ is trivial, $\varphi$ is identically zero on $R^{\prime}$. Thus $\wedge^{2} \varphi$ vanishes to order two at $R_{k} \cap R_{k+1}$ and hence vanishes on $R_{k}$. Thus $\wedge^{2} \varphi$ vanishes at $P_{1}$.

Third, we claim that if $\operatorname{deg} E_{R}^{\prime} \leqslant 1$ and $\varphi$ has rank one at $P_{1}$ and $P_{2}$, then there is quotient line bundle $L$ of $E_{R}^{\prime}$ so that $\varphi\left(E_{R}\right)$ maps to zero in $L$. Indeed, $\wedge^{2} \varphi$ must be zero since $\wedge^{2} E_{R}^{\prime}$ has positive degree on at most one component $R_{l}$ and its degree on that component is one. Since $\wedge^{2} \varphi$ vanishes at $P_{1}$ and $P_{2}$, we see $\wedge^{2} \varphi=0$. Now $\varphi$ factors through a subline bundle of $E_{R}^{\prime}$ unless $\varphi$ vanishes at some node $R_{i} \cap R_{i+1}$. But then the components $\varphi_{p q}$ vanish at this node. We may assume $l \leqslant i$. Then $E_{R_{j}}^{\prime}$ is trivial for $j \geqslant i$ so $\varphi_{p q}$ vanishes at $P_{2}$ and hence $\varphi$ vanishes at $P_{2}$. This contradicts our assumption.

Fourthly, if $\tilde{E}$ and $\tilde{E}^{\prime}$ have the same degree, we claim $\tilde{\varphi}$ cannot vanish at $P_{1}$ and $P_{2}$. Suppose not. Our claim is clear if $\tilde{E}$ and $\tilde{E}^{\prime}$ are semistable. We have a nonzero homomorphism $\tilde{\varphi}$ from $\tilde{E}$ to $\tilde{E}^{\prime}\left(-P_{1}-P_{2}\right)$. Now $\wedge^{2} \tilde{\varphi}$ vanishes, since $\operatorname{deg} \tilde{E}>\operatorname{deg} \tilde{E}^{\prime}\left(-P_{1}-P_{2}\right)$. Hence $\varphi$ factors through a subline bundle $L$ of $\tilde{E}^{\prime}\left(-P_{1}-P_{2}\right)$. But $L$ must have degree at least $\alpha$, since $\tilde{E}$ is at most slightly unstable. But $\operatorname{deg} L \leqslant \alpha-1$, since $\tilde{E}^{\prime}$ is at most slightly unstable. Hence $\tilde{\varphi}$ is zero and hence $\varphi$ is zero by the first claim.

Fifthly, we claim that if $\operatorname{deg} \tilde{E}>\operatorname{deg} \tilde{E}^{\prime}$ then $\tilde{E}$ is slightly unstable and $\tilde{E}^{\prime}$ is strictly semistable. Further, $\tilde{\varphi}$ has rank one at $P_{1}$ and $P_{2}$. In fact, $\tilde{\varphi}$ must factor through the destabilizing quotient of $\tilde{E}$, which must be a subbundle of $\tilde{E}^{\prime}$.

We lastly claim $\varphi$ is an isomorphism. Indeed suppose first that $\operatorname{deg} \tilde{E}>$ $\operatorname{deg} \tilde{E}^{\prime}$. Then from our fifth claim we see $\operatorname{deg} E_{R}=0$ and $\operatorname{deg} E_{R}^{\prime}=1$. Our third claim shows there is quotient line bundle of $E_{R}^{\prime}$ which coincides with coker $\tilde{\varphi}$ over $P_{1}$ and $P_{2}$. Hence there is a quotient line bundle $L$ of $E^{\prime}$ so that $\varphi(E)$ maps to zero in $L$. Let $M=\operatorname{Ker}\left(E^{\prime}, L\right)$. Then $\operatorname{deg} M \leqslant \alpha$, since $E^{\prime}$ is weakly stable. But the map of $\tilde{E}$ to $\tilde{M}$ factors through the destabilizing quotient of $\tilde{E}$, so $\operatorname{deg} \tilde{M}>\alpha$. Since $\varphi_{R}$ has rank one on all the $R_{i} \cap R_{i+1}$ and $\tilde{E}_{R}=\vartheta_{R} \oplus \vartheta_{R}$, we see $\operatorname{deg} \tilde{M}_{R} \geqslant 0$. Hence $\operatorname{deg} \tilde{M}_{R}=0$ and $\operatorname{deg} \tilde{M}=\alpha$. Further $M$ is a quotient of $E$, so $\operatorname{deg} M \geqslant \alpha+1$ since $E$ is weakly stable.

Thus we may assume $\operatorname{deg} \tilde{E}=\operatorname{deg} \tilde{E}^{\prime}$. Now $\wedge^{2} \tilde{\varphi}=0$, since if $\wedge^{2} \tilde{\varphi} \neq 0$, then $\tilde{\varphi}$ is an isomorphism since $\operatorname{deg} \tilde{E}=\operatorname{deg} \tilde{E}^{\prime}$. Hence $\varphi$ would be an isomorphism. Now if $\wedge^{2} \tilde{\varphi}=0$, either $\tilde{E}$ or $\tilde{E}^{\prime}$ must be unstable, since any nonzero 
map between two stable bundles of the same degree and rank is an isomorphism. Hence $\operatorname{deg} E_{R}^{\prime} \leqslant 1$. If $\operatorname{deg} E_{R}^{\prime}=1$, then $\tilde{E}$ and $\tilde{E}^{\prime}$ are semistable and $\tilde{\varphi}$ has rank one at $P_{1}$ and $P_{2}$. One sees as above that $\varphi$ factors through a subbundle $M$ of $E^{\prime}$ which is a quotient of $E$. This contradicts the weak stability of $E$ and $E^{\prime}$. Finally, suppose $\operatorname{deg} E_{R}^{\prime}=0$. If $\varphi_{R}$ vanished at $P_{1}$, it would also vanish at $P_{2}$ and vice versa, since $E_{R}$ and $E_{R}^{\prime}$ are trivial. Hence $\varphi$ has rank one at $P_{1}$ and $P_{2}$. This again contradicts the weak stability of $E$ and $E^{\prime}$. $k$.

Corollary of proof 3.2. If $E$ is potentially stable on $X_{n}$, then $\operatorname{Hom}(E, E)=$

4. Let $C$ be a smooth curve and let $t P \in C$. Let $\pi$ : $\mathcal{X} \rightarrow C$ be a flat family of curves with $\mathcal{X}$ smooth over $k$ and $\pi^{-1}(P)=\mathcal{X}_{P}=X_{0}$, where $X_{0}$ is our nodal curve. We assume $\pi$ has a section and that $\pi$ is smooth away from $P$. Now let $F(T)$ be the set of closed subschemes $Y \subseteq\left(\mathscr{X} \times{ }_{C} T\right) \times_{k} G$ which have the following properties:

(i) $Y$ is a flat family of curves of genus $g$ over $T$.

(ii) The induced map from $Y$ to $T \times_{k} G$ is an embedding and for each closed $t \in T$, the corresponding curve in $G$ is Hilbert semistable.

(iii) Locally on $T$, there is an isomorphism between the relative dualizing sheaf $\omega_{Y / T}$ and the pullback of $\omega_{X / C}$.

We will see (iii) is an open condition. Assuming this, we see $F$ is an open subfunctor of the relative Hilbert scheme of $\chi X G$, and so $F$ is representable by a scheme $p: \mathcal{Y} \rightarrow C$. First notice that a closed point of $\mathcal{Y}$ lying over $P$ consists of a curve $X^{\prime} \subseteq G$ which is $m$-Hilbert semistable and a map $\varphi$ of $X^{\prime}$ to $X_{0}$ so that the pullback of $\omega_{X}$ is $\omega_{X^{\prime}}$. Since the genus of $X_{0}$ and $X^{\prime}$ are the same, one sees that $X^{\prime}$ is $X_{n}, 0 \leqslant n \leqslant 2$, and the map from $X^{\prime}$ to $X_{0}$ is the map which collapses the $R_{i}$ to the unique node of $X_{0}$.

Proposition 4.1. $\mathcal{Y}$ is smooth over $k$ and $\mathcal{Y}_{P}$ is a reduced divisor with normal crossings. Further $S L(W)$ operates freely on $\mathcal{Y}$.

Before proving the proposition, we need to study deformations of $X_{n}$. Let $A$ be an artinian $k$ algebra. Then Schessingers's theory shows that given a flat deformation $Z$ of $X_{n}$ over $\operatorname{Spec} A$, there are $a_{1}, \cdots, a_{n} \in A$ so that at the $i$ th node $N_{i}=R_{i} \cap R_{i+1}$, we have $\hat{\theta}_{Z, N_{i}} \cong A[[x, y]] /\left(x y-a_{i}\right)$. The $a_{i}$ are determined up to a unit, so we refer to $a_{i}=0$ as the equation of the $i$ th node. If $Z^{\prime}$ is another deformation of $X_{n}$ so that $a_{i}$ is the equation of the $i$ th node of $Z^{\prime}$, then $Z$ and $Z^{\prime}$ are locally isomorphic in the Zariski topology.

Now let $W=\operatorname{Spec} k\left[\left[t_{1}, \cdots, t_{n}\right]\right]$. We choose $t$ to be a uniformizing parameter in $\Theta_{C, P}$, and we map $W$ to $C$ so that $t=t_{1} \cdots t_{n}$.

Lemma 4.2. There is a deformation $Z$ of $X_{n}$ over $W$ and a morphism $\psi$ : $Z \rightarrow \mathfrak{X} \times_{C} W$ so that $\psi^{*}\left(\omega_{\mathcal{X} / C}\right) \cong \omega_{Z / W}$ and so that $t_{i}=0$ is the equation for the ith node of $X_{n}$. 
Proof. Consider the case $n=1$. There is an étale cover $q: Y_{0} \rightarrow X_{0}$ so that $Y_{0}$ consists of two copies of $X$ with $P_{1}$ on copy one glued to $P_{2}$ on copy two and vice versa. Let $\hat{X}$ be the completion of $\mathcal{X}$ at $X_{0}$. Then we can find an étale cover $q: \hat{Y} \rightarrow \hat{X}$ reducing to $Y_{0} \rightarrow X_{0}$ over $P$. Now let $Q_{1}$ and $Q_{2}$ be the two nodes of $Y_{0}$. We can find local parameters $x$ and $y$ at $Q_{1}$ so $t=x y$. Thus in some neighborhood of $Q_{1} \in \hat{Y} \times \hat{c} W$, we have $x y=t_{1} t_{2}$. Let $\Phi_{1}$ be the ideal generated by $x$ and $t_{1}$. One checks $\Phi_{1}$ is a Cartier divisor away from $Q_{1}$. Let $\Phi_{2}=i^{*}\left(\Phi_{1}\right)$, where $i$ is the involution on $\hat{Y}$. Now blow up $\hat{Y}$ at $q_{1}$ around $Q_{1}$ and $\Phi_{2}$ around $Q_{2}$ to obtain $\hat{Y}^{\prime}$. Since $\Phi_{1}$ and $\Phi_{2}$ are Cartier away from $Q_{1}$ and $Q_{2}$, this operation is well defined. Now $i$ still acts on $\hat{Y}^{\prime}$ as a fixed point free automorphism, so we can form the quotient $Z$ by dividing $\hat{Y}^{\prime}$ by the action of $i$.

We can check the local behavior of $\hat{Y}^{\prime}$. Let

$$
R=k\left[\left[x, y, t_{1}, t_{2}\right]\right] /\left(x y-t_{1} t_{2}\right)
$$

and consider $R_{1}=R\left[x / t_{1}\right]$ and $R_{2}=R\left[t_{1} / x\right]$. Let $m$ be a maximal ideal in $R_{1}$. Let $a$ be the residue class of $x$ in $R_{1} / m$ and let $x^{\prime}=x / t_{1}$. If $a \neq 0$, then $x^{\prime}-a, t_{1}$ and $t_{2}$ generate $m$ since $y=t_{2} / x^{\prime}$. Thus $R_{1}$ is regular at such a point and the equations $t_{1}=t_{2}=0$ define a smooth curve. If $x^{\prime} \in m$, then we have the relation $t_{2}=x^{\prime} y$, so $x^{\prime}, y$ and $t_{1}$ generate $m$. Thus $R_{1}$ is regular at such a point and $t_{2}$ is the equation for the node. Now since $t_{1} / x=y / t_{2}$ we see $R_{2}=R\left[y / t_{2}\right]$ and so $t_{1}$ is the equation for the node $y / t_{2}=x=t_{1}=0$. So the two nodes of $Z$ have equation $t_{1}=0$ and $t_{2}=0$. Similarly, one can check locally $\psi^{*}\left(\omega_{\mathscr{X} / C}\right)=\omega_{Z / W}$.

The case of $n>1$ is handled similarly.

Now let $\pi: X_{n} \rightarrow X_{0}$ be the standard map. There is a map from $\pi^{*} \Omega_{X_{0}}^{1}$ to $\Omega_{X_{n}}^{1}$ and hence a map

$$
\varphi: \operatorname{Hom}\left(\Omega_{X_{n}}^{1}, \vartheta\right) \rightarrow \operatorname{Hom}\left(\pi^{*} \Omega_{X_{0}}^{1}, \vartheta\right)
$$

Let $E$ be the union of the $R_{i}$ 's in $X_{n}$.

Lemma 4.3. $\operatorname{Ker} \varphi \cong \oplus \vartheta_{R_{i}}$, Coker $\varphi \subseteq \vartheta_{E}\left(-P_{1}\right) \oplus \vartheta_{E}\left(-P_{2}\right)$.

Proof. We have a (nonexact) sequence of maps over some étale neighborhood of $E$,

$$
\vartheta \oplus \Theta \stackrel{\varphi_{3}}{\rightarrow} \pi^{*} \Omega_{X_{0}}^{1} \rightarrow \Omega_{X_{n}}^{1} \rightarrow \Omega_{E}^{1}
$$

To define $\varphi_{3}$, we let the node of $X_{0}$ be given by $x y=0$. Then $\varphi_{3}(f, g)=f d x$ $+g d y$. Dualizing $\varphi_{3}$, we obtain a map

$$
\psi_{3}^{\prime}: \operatorname{Hom}\left(\pi^{*} \Omega^{1}, \theta\right) \rightarrow \theta \oplus \theta .
$$

Let

$$
\psi_{3}^{\prime \prime}: \vartheta \oplus \theta \rightarrow \vartheta_{E} \oplus \vartheta_{E}
$$


be the natural map and let $\psi_{3}=\psi_{3}^{\prime \prime} \circ \psi_{3}^{\prime}$. Then dualizing (4.3.1), we have

$$
0 \rightarrow \operatorname{Hom}\left(\Omega_{E}^{1}, \mathcal{\theta}\right) \stackrel{\psi_{1}}{\rightarrow} \operatorname{Hom}\left(\Omega_{X_{n}}^{1}, \mathcal{\theta}\right) \stackrel{\psi_{2}}{\rightarrow} \operatorname{Hom}\left(\pi^{*} \Omega_{X_{0}}^{1}, \mathcal{\theta}\right) \stackrel{\psi_{3}}{\rightarrow} \vartheta_{E} \oplus \vartheta_{E}
$$

We claim (4.3.2) is exact and that im $\psi_{3} \subseteq \vartheta_{E}\left(-P_{1}\right) \oplus \mathcal{\vartheta}_{E}\left(-P_{2}\right)$. Our claim is readily verified except at $P_{1}$ and $P_{2}$. So consider $P_{1} \in X_{n}$. We can introduce a local parameter $y^{\prime}$ at $P_{1}$ so that $X_{n}$ is defined by $x y^{\prime}=0$ near $P_{1}$. Further, $y=0$ near $P_{1}$. Now let $e_{1}=\pi^{*}(d x)$ and $e_{2}=\pi^{*}(d y)$. Near $P_{1}, \pi^{*} \Omega_{X_{0}}^{1}$ is generated by $e_{1}$ and $e_{2}$ subject to $x e_{2}=0$. Near $P_{1}, \Omega_{X_{n}}^{1}$ is generated by $e_{1}$ and $d y^{\prime}$ subject to $x d y^{\prime}+y^{\prime} e_{1}=0$. Further, $e_{2}$ maps to zero in $\Omega_{X_{n}}^{1}$ at $P_{1}$. Let us check the image of $\psi_{3}$ is contained in $\theta\left(-P_{1}\right) \oplus \theta\left(-P_{2}\right)$. Let $\Lambda \in \operatorname{Hom}\left(\pi^{*} \Omega_{X_{0}}^{1}, \theta\right)$ be defined near $P_{1}$. Then $x \Lambda(d y)=0$, so $\Lambda(d y)$ must vanish on the curve $y^{\prime}=0$. Hence $\Lambda(d y)$ vanishes at $P_{1}$. Thus the image of $\psi_{3}$ is contained in $\vartheta_{E}\left(-P_{1}\right) \oplus \vartheta_{E}$ near $P_{1}$. Near $P_{2}$, the image is contained in $\vartheta_{E} \oplus \vartheta_{E}\left(-P_{2}\right)$. Our other assertions may be similarly verified. Further, $\operatorname{Hom}\left(\Omega_{E}^{1}, \theta\right)=\oplus \vartheta_{R_{i}}$.

Corollary 4.4. The natural map

$$
\psi: H^{1}\left(\operatorname{Hom}\left(\Omega_{X_{n}}^{1}, \theta\right)\right) \rightarrow H^{1}\left(\operatorname{Hom}\left(\pi^{*}\left(\Omega_{X_{0}}^{1}\right), \theta\right)\right)
$$

is injective.

Proof. $\quad H^{1}\left(\oplus \vartheta_{R_{i}}\right)=0$ and $H^{0}\left(\vartheta_{E}\left(-P_{1}\right) \oplus \vartheta_{E}\left(-P_{2}\right)\right)=0$.

Let $A$ be an artin local $k$ algebra, let $T=\operatorname{Spec} A$ and suppose $T$ is a $C$ scheme with the closed point of $T$ going to $R$. Let $t$ be a uniformizing parameter in $\theta_{C, R}$.

Proposition 4.5. Suppose $Z^{\prime}$ is a flat deformation of $X_{n}$ over $T$ and that there is a $T$ morphism $\psi^{\prime}$ of $Z^{\prime}$ to $\chi \times_{C} T$ which reduces to the standard morphism of $X_{n}$ to $X_{0}$. Then there is a map of $T$ to $W$ so that $Z^{\prime}$ and $\psi^{\prime}$ are isomorphic to the pullbacks of the $Z$ and $\psi$ of Lemma 4.2. Further, if $a_{i}=0$ is the equation of the ith node of $Z^{\prime}$, then $\prod_{i} a_{i}$ generates the ideal $(t)$ in $A$.

Proof. We may assume that $A$ has a principal ideal $(\varepsilon)$ of $k$-dimension 1 and that the proposition is true for $A_{0}=A /(\varepsilon)$. Let $T_{0}=\operatorname{Spec} A_{0}$.

We can find a map $\varphi_{1}$ of $T$ to $W$ so that the pullback $\left(Z_{1}^{\prime \prime}, \psi_{1}^{\prime \prime}\right)$ of $(Z, \psi)$ is isomorphic to $\left(Z^{\prime}, \psi^{\prime}\right)$ over $T_{0}$ and so that the equations for the $i$ th nodes of $Z_{1}^{\prime \prime}$ and $Z^{\prime}$ agree. Thus $Z^{\prime}$ and $Z_{1}^{\prime \prime}$ are locally isomorphic over $T$. Choose an affine open cover $U_{i}$ of $X_{n}$ and let $U_{i}^{\prime}$ and $U_{i}^{\prime \prime}$ be the corresponding open covers of $Z^{\prime}$ and $Z^{\prime \prime}$. We may assume there are isomorphisms $\Phi_{i}: \mathcal{\theta}_{U_{i}^{\prime}} \rightarrow \mathcal{O}_{U_{i}^{\prime \prime}}$ which agree over $T_{0}=\operatorname{Spec} A_{0}$. Now on $U_{i}^{\prime} \cap U_{j}^{\prime}$, we have

$$
\Phi_{i}(f)=\Phi_{j}(f)+\varepsilon \lambda_{i j}(d f)
$$

for all $f \in \Gamma\left(U_{i}^{\prime}, \theta\right)$ and some $\lambda_{i j} \in \Gamma\left(U_{i} \cap U_{j}, \operatorname{Hom}\left(\Omega_{X_{n}}^{1}, \theta\right)\right)$. As usual, $\lambda_{i j}$ is a cocycle and $Z^{\prime}$ and $Z_{1}^{\prime \prime}$ are isomorphic over $C$ if and only if $\left\{\lambda_{i j}\right\}$ is a 
coboundary. Let $\mu_{i j}$ be the image of $\lambda_{i j}$ in $\Gamma\left(U_{i} \cap U_{j}, \operatorname{Hom}\left(\pi^{*} \Omega_{X_{0}}^{1}, \theta\right)\right)$, and let $\mu$ be the corresponding cohomology class in $H^{1}\left(\operatorname{Hom}\left(\pi^{*} \Omega_{X_{0}}^{1}, \theta\right)\right)$. But $\mu$ is just the obstruction to extending the map from $Z_{0}^{\prime}$ to $\mathcal{X} \times_{C} T_{0}$ to a $C$ morphism from $Z^{\prime}$ to $\mathcal{X} \times{ }_{C} T$. Corollary 4.5 shows $\lambda_{i j}$ is a coboundary.

Next we study the action of $\operatorname{SL}(W)$ on the Hilbert scheme of $G$.

Lemma 4.6. Suppose $\lambda: X_{n} \rightarrow G$ is an embedding with $n \geqslant 1$ and $\lambda\left(X_{n}\right)$ is a potentially stable curve. Suppose $\sigma \in S L(W)$ fixes $\lambda\left(X_{n}\right)$ and is the identity on $X \subseteq X_{n}$. Then $\sigma$ is a multiple of the identity.

Proof. Let $E=\lambda^{*}(\mathcal{E})$. The map $\lambda$ is given by choosing a basis $\left\{s_{i}\right\}$ of $H^{0}\left(X_{n}, E\right)$ and $\sigma \circ \lambda$ is obtained by a basis $\left\{s_{i}^{\prime}\right\}$ of $H^{0}\left(X_{n}, E\right)$. Since $\sigma$ induces the identity on $X$, we have that $s_{i}=s_{i}^{\prime}$ on $X$ up to a scalar multiple. But $H^{0}\left(X_{n}, E\right) \rightarrow H^{0}(X, E)$ is injective since $E$ is potentially stable. Further, since $W=H^{0}(X, E)$, we see $\sigma$ is the identity up to a scalar multiple.

With the same notation as in Lemma 4.6, we have

Lemma 4.7. Let $D$ be a vector field on $G$ which is tangent to $\lambda\left(X_{n}\right)$. Then $D$ is zero.

Proof. Let $k[\varepsilon]=k[x] /\left(x^{2}\right)$. If $V$ is a scheme over $k$, we let $V[\varepsilon]=V$ $\times{ }_{k} \operatorname{Spec} k[\varepsilon]$. Now $D$ induces a $k[\varepsilon]$ map from $G[\varepsilon]$ to $G[\varepsilon]$. Note that $D$ is zero on $\lambda\left(P_{1}\right)$ and $\lambda\left(P_{2}\right)$ since $D$ is tangent to $\lambda\left(X_{n}\right)$. Further, $D$ actually induces a map of $\lambda\left(X_{n}\right)[\varepsilon]$, and hence we may regard $D: X_{n}[\varepsilon] \rightarrow X_{n}[\varepsilon]$. Now there are no vector fields on $X$ vanishing at $P_{1}$ and $P_{2}$, so $D$ vanishes on $X \subseteq X_{n}$. Now the map from $G[\varepsilon]$ to $G[\varepsilon]$ is just given by a new basis $S_{1}^{\prime}, \cdots, S_{n}^{\prime}$ of $H^{0}(G, \mathcal{E})[\varepsilon]$ which coincides with the old basis $S_{1}, \cdots, S_{n} \bmod \varepsilon$. Since $D$ is zero on $X \subseteq X_{n}$, we have that $S_{i}=S_{i}^{\prime}$ on $X[\varepsilon]$ up to a scalar multiple. We may assume $S_{i}=S_{i}^{\prime}$ on $X[\varepsilon]$. But the map from $H^{0}\left(X_{n}[\varepsilon], \mathcal{E}\right)$ to $H^{0}(X[\varepsilon], \mathcal{E})$ is injective, so the $S_{i}=S_{i}^{\prime}$ in $H^{0}\left(X_{n}[\varepsilon], \mathcal{E}\right)$. Hence $S_{i}=S_{i}^{\prime}$ in $H^{0}(G[\varepsilon], \mathcal{E})$ and so $D=0$.

With the notation of Lemma 4.6 again, we have

Lemma 4.8. Let 9 be the ideal sheaf of $\lambda\left(X_{n}\right)$. Then the natural map

$$
p: H^{0}\left(G, T_{G}\right) \rightarrow \operatorname{Hom}\left(g / g^{2}, \Theta_{\lambda\left(X_{n}\right)}\right)
$$

is injective.

Proof. Identify $\lambda\left(X_{n}\right)$ and $X_{n}$. First note that the map from $H^{0}\left(G, T_{G}\right)$ to $H^{0}\left(X_{n}, T_{G} \otimes \vartheta_{X_{n}}\right)$ is injective. Indeed on $G$, we have a tautological exact sequence

$$
0 \rightarrow \mathscr{g} \rightarrow \mathcal{\theta}^{r} \rightarrow \mathcal{E} \rightarrow 0
$$

where we denote $\operatorname{dim} W$ by $r$ instead of by $n$ as above. Now $T_{G}=\operatorname{Hom}(\mathcal{g}, \mathcal{E})$, so we have an exact sequence

$$
0 \rightarrow \operatorname{Hom}(\mathcal{E}, \mathcal{E}) \rightarrow \mathcal{E}^{r} \rightarrow T_{G} \rightarrow 0 .
$$


Thus we have the commutative diagram with exact rows:

$$
\begin{aligned}
& 0 \longrightarrow H^{0}(G, \operatorname{Hom}(\mathcal{E}, \mathcal{E})) \longrightarrow H^{0}(G, \mathcal{E})^{r} \longrightarrow H^{0}\left(G, T_{G}\right) \longrightarrow 0 \\
& \downarrow \alpha \quad \downarrow \beta \quad \downarrow \gamma \\
& 0 \longrightarrow H^{0}\left(X_{n}, \operatorname{Hom}(\mathcal{E}, \mathcal{E})_{X_{n}}\right) \longrightarrow H^{0}\left(X_{n}, \mathcal{E}_{X_{n}}\right)^{r} \longrightarrow H^{0}\left(X_{n},\left(T_{G}\right)_{X_{n}}\right)
\end{aligned}
$$

Now $\alpha$ is an isomorphism, since $\operatorname{Hom}\left(\mathcal{E}_{X_{n}}, \mathcal{E}_{X_{n}}\right)=k$. Further, $\beta$ is an isomorphism. So $\gamma$ is injective. Lemma 4.8 shows $(\operatorname{im} \gamma) \cap H^{0}\left(X, T_{X}\right)=0$. On the other hand, from the exact sequence

$$
0 \rightarrow g / \mathscr{I}^{2} \rightarrow\left(\Omega_{E}^{1}\right)_{X_{n}} \rightarrow \Omega_{X_{n}}^{1} \rightarrow 0
$$

we see that the following is exact:

$$
0 \rightarrow H^{0}\left(X_{n}, T_{X_{n}}\right) \rightarrow H^{0}\left(X_{n}, T_{G}\right) \rightarrow \operatorname{Hom}\left(q / \mathscr{G}^{2}, \Theta_{X_{n}}\right) .
$$

So our claim follows.

Proof of Proposition 4.1. Suppose $A$ is an artinian local $k$ algebra with an ideal $(\varepsilon)$ of dimension one. Let $A_{0}=A /(\varepsilon), T=\operatorname{Spec} A$ and $T_{0}=\operatorname{Spec} A_{0}$. To show $\mathscr{Y}$ is smooth, it suffices to show that $F(T)$ surjects to $F\left(T_{0}\right)$. Let $Y_{0} \subseteq\left(\mathcal{X} \times_{X} T_{0}\right) \times_{k} G$ be an element of $F\left(A_{0}\right)$. Consider $Y_{0}$ as an abstract deformation of $X_{n}$ mapping to $\mathcal{X} \times_{C} T_{0}$. Then by Proposition 4.5 there is an extension $Y$ of $Y_{0}$ over $T$ and a map of $T$ to $C$ so that $Y$ maps to $\mathcal{X} \times_{C} T$. Further, $\mathcal{E}_{0}=\mathcal{E}_{Y_{0}}$ can be extended to a bundle $\mathcal{G}$ on $Y$, since the obstruction to lifting $\mathcal{E}_{0}$ lies in $H^{2}\left(\operatorname{Hom}(\mathcal{E}, \mathcal{E})_{X_{n}}\right)$. Finally, the sections of $\mathcal{E}_{0}$ defining the given map of $Y_{0}$ to $G$ can be extended to $\mathscr{G}$, since $H^{1}\left(X_{n}, \mathcal{E}_{X_{n}}\right)=0$. Thus $Y$ is smooth over $k$.

Consider $\mathcal{Y}_{P}$ as a Cartier divisor defined by $t=0$. Let $R$ be a closed point of $\mathcal{Y}_{P}$. Now over $T=\operatorname{Spec} \hat{\theta}_{\mathscr{Y}, R}$ there is a universal family of curves $Y \subseteq$ $\left(\mathcal{X} \times_{C} T\right) \times_{k} G$. Let $z_{i} \in \hat{\mathcal{O}}_{\mathscr{Y}, R}$ be the equation for the $i$ th node of $Y$. Then $\Pi z_{i}$ generates $t$ (Proposition 4.5). On the other hand, for each $i$ if $T_{0}=$ Spec $k[\varepsilon] /\left(\varepsilon^{2}\right)$, we can find $Y_{i} \in F\left(T_{0}\right)$ mapping to $R$, so that if $a_{i j}$ is the equation for the $j$ th node on $Y_{i}$, then $a_{i j}=\delta_{i j}$. Hence the $d z_{i}$ are independent, and $\mathcal{Y}_{P}$ has normal crossings on $\mathcal{Y}$.

Let $S_{g, d}$ be the Hilbert scheme of curves of genus $g$ and degree $d$ in $G$ and let $U \subseteq S_{g, d}$ be the set of semistable points. Since $g \geqslant 2$, there are at most finitely many maps $\sigma$ of a semistable curve to its stable model. Thus the induced map

$$
\varphi: \mathcal{Y} \rightarrow U \times_{k} C
$$

is quasifinite. We claim $\varphi$ is finite. Suppose not. Then we can find a smooth curve $S$, a $R \in S$ and a map $\psi: S-R \rightarrow \mathcal{Y}$ which induces a map $\psi^{\prime}$ of $S$ to $U \times C$. We consider first the case when $S-R$ maps to $C-P$ and $R$ maps to $P$. Using an argument similar to that used in Proposition 3.1, we see that the 
family of semistable curves induced by $\psi^{\prime}$ over $S$ maps to the family of stable curves $\mathcal{X} \times{ }_{C} S$. But then $\psi$ can be defined at $R$. The other cases can be similarly handled, so $\varphi$ is finite.

$\mathrm{SL}(W)$ acts on $U \times C$ and on $\mathcal{Y}$ over $k$. We can cover $U$ by invariant affines $U_{i}$ on which $\operatorname{SL}(W)$ acts properly and so cover $Y$ by invariant affines $V_{i}=\varphi^{-1}\left(U_{i} \times C\right)$ on which $\mathrm{SL}(W)$, acts properly. We claim $\mathrm{SL}(W)$ acts freely. Lemma 4.6 shows that $\operatorname{SL}(W)$ has no fixed points. Indeed, if $\operatorname{SL}(W)$ fixed a closed point of $\mathscr{Y}$, it would induce a nontrivial automorphism of $X \subseteq X_{n}$. But then if $p$ is a standard map of $X_{n}$ to $X_{0}$, we would have $\sigma \circ p \neq p$. Thus $\sigma$ has no fixed points. Further, Lemma 4.8 shows the map of the Lie algebra of $\mathrm{SL}(W)$ to the tangent space of the Hilbert scheme at a given closed point is injective. So our claim is established.

So a geometric quotient $\mathscr{W}$ of $\mathcal{Y}$ by $\operatorname{SL}(W)$ exists [3, Proposition 3.12]. Further, $\mathcal{Y}$ is a principal $\operatorname{SL}(W)$ bundle over $\mathscr{W}[1$, Proposition 0.9]. Since $\mathcal{Y}$ is smooth, W is also smooth. Further, $W_{P}$ has normal crossings on $W$. We finally claim that $\mathcal{W}$ is projective over $C$. If $\mathcal{W}$ is not proper over $C$, we can find a $C$ curve $S$ and a morphism $\psi$ of $S-R$ to $\mathcal{W}$. By passing to a ramified cover of $S$, we can assume $\psi$ can be lifted to a map $\psi^{\prime}$ of $S-R$ to $\mathcal{Y}$ so that $\psi^{\prime}$ induces a map of $S$ to $S_{g, d}$. We may further assume that $\psi^{\prime}(R)$ is a semistable point $Q$ of $S_{g, d}$ and that if $Q$ is strictly semistable, then the automorphism group of $Q$ as a curve on $G$ is infinite. By the results of [1], $Q$ as an abstract curve is semistable and hence is either smooth or one of the $X_{n}$. By Corollary 3.2, $Q$ has no continuous families of automorphisms as a curve in $G$. Thus $Q \in U$ and $\psi^{\prime}$ factors through $U$. Since $\varphi$ is finite, $\psi^{\prime}$ factors through $\mathcal{Y}$.

Now let $T$ be a $C$ scheme and let $G(T)$ consist of triples $(Y, E, q)$ where $Y$ is a semistable family of curves, of genus $g$ over $T, q: Y \rightarrow \mathfrak{X} \times{ }_{C} T$ satisfies (iii) above, and $E$ is potentially stable on all the fibers of $p: Y \rightarrow T$. Then any element of $G(T)$ can be lifted locally to $F(T)$. We thus get a map of the functor $G$ to ข which is bijective on closed points.

5. Let $S$ be a smooth curve and let $R \in S$ be fixed. Let $E^{\prime}$ and $E^{\prime \prime}$ be bundles on $S$. We define a rational isomorphism $\varphi$ of $E^{\prime}$ to $E^{\prime \prime}$ to be an isomorphism of $E^{\prime}$ with $E^{\prime \prime}$ over the generic point of $S$. There is a unique $r \in \mathbf{Z}$ such that $\varphi$ induces a map

$$
\varphi^{\prime}: E^{\prime}(r R) \rightarrow E^{\prime \prime}
$$

which is defined (i.e., holomorphic) and nonzero at $R$. There is a unique $s$ so that $\left(\operatorname{coker} \varphi^{\prime}\right)_{R}=\vartheta_{R} / m^{s}$, where we define $m^{0}=\vartheta_{R}$. We say $(r, s)$ is the type of $\varphi$ with respect to $E$ and $E^{\prime}$.

Suppose $E$ is a vector bundle on $X \times S$ and $\varphi$ is an isomorphism of $\left(E_{P_{1}}\right)_{U}$ with $\left(E_{P_{2}}\right)_{U}$ where $U=S-R$. We can use $\varphi$ as descent data to form a bundle 
$E_{0}$ on $X_{0} \times U$. Our aim in this section is to define a semistable family of curves $X^{\prime}$ and a bundle $E^{\prime}$ on $X^{\prime}$ extending $X_{0} \times U$ and $E_{0}$ for certain types of $\varphi$. The resulting family will be called the geometric realization of $\varphi$.

First, if $\varphi$ has type $(r, s)$, then $\varphi^{-1}$ has type $(-r-s, s)$. Indeed, notice that $Q_{2}=\operatorname{coker} \varphi^{\prime}$ is an $\theta_{R} / m^{s}$ quotient of $E_{P_{2}}$. We will, in general, feel free to localize around $R$ without comment. Thus we can assume that $E_{P_{2}}$ is a direct sum $M_{1} \oplus M_{2}$ where $M_{2}$ coincides with $Q_{2}$ over $\operatorname{Spec} \vartheta_{R} / m^{s}$. Thus

$$
\operatorname{Ker}\left(E_{P_{2}} \rightarrow Q_{2}\right)=M_{1} \oplus M_{2}(-s R) \text {. }
$$

Let $N_{1} \oplus N_{2}$ be a direct sum decomposition of $E_{P_{1}}$ which corresponds to $M_{1} \oplus M_{2}(-s R)$ under the isomorphism of $E_{P_{1}}(r R)$ with $\operatorname{Ker}\left(E_{P_{2}}, Q_{2}\right)$. Thus we have isomorphisms

$$
\varphi_{1}: N_{1}(r R) \rightarrow M_{1}, \quad \varphi_{2}: N_{2}(r R) \rightarrow M_{2}(-s R) .
$$

Thus $M_{i}((-s-r) R)$ maps to $N_{i}$ and this map is nonzero at $R$ for $i=2$. Further, $N_{1} / \varphi_{1}^{-1}\left(M_{1}((-r-s) R)\right)=\theta_{R} / m^{s}$, so $\varphi^{-1}$ has type $(-r-s, s)$.

Case $(0,1)$. We suppose $\varphi$ has type $(0,1)$. This means that $\varphi: E_{P_{1}} \rightarrow$ $\operatorname{Ker}\left(E_{P_{2}}, L_{2}\right)$, where $L_{2}$ is an $R$ quotient of $E_{P_{2}}$. We let $L_{1}$ be the $R$ quotient of $E_{P_{1}}$ corresponding to the canonical quotient of $\operatorname{Ker}\left(E_{P_{1}}, L_{2}\right)$. On $X \times S$ blow up the point $P_{2} \times R$ to obtain a surface $p: \mathscr{X}_{1} \rightarrow X \times S$. Call the exceptional divisor $D_{2}$, and let the proper transform of $P_{i} \times S$ be $H_{i}$. Denote the proper transform of $X \times R$ by $X$.

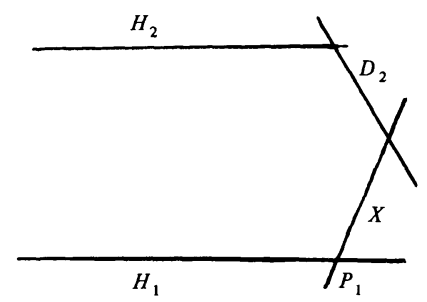

Now let $E^{(2)}=p^{*}(E)$. Note that $p^{*}\left(L_{2}\right)$ is a $D_{2}$ quotient of rank one. Let $E^{\prime}=\operatorname{Ker}\left(E^{(2)}, p^{*}\left(L_{2}\right)\right)$. The map $\varphi$ from $E_{H_{2}}^{\prime}$ to $\operatorname{Ker}\left(E_{P_{2}}, L_{2}\right)$ is an isomorphism. To obtain the geometric realization of $\varphi$, we glue $H_{1}$ to $H_{2}$ and use $\varphi$ as an isomorphism of $E_{H_{1}}^{\prime}$ to $E_{H_{2}}^{\prime}$. We have

(5.1.1) $E_{D_{2}}^{\prime}$ is standard of type 1 .

(5.1.2) $E_{X}^{\prime}$ is the modification of $E_{X \times R}$ at $L_{2}$.

(5.1.3) At $P_{1}$, the standard quotient $\theta$ of $E_{D_{2}}^{\prime}$ is glued to $L_{1^{\prime}}$ the $P_{1} \times R$ quotient of $E_{P_{1}}^{\prime}=E_{P_{1}}$.

(5.1.4) At $P_{2}$, the standard quotient $\theta$ of $E_{D_{2}}^{\prime}$ is glued to the canonical quotient of $E_{X}^{\prime}$ from (5.1.2). 
Indeed, (5.1.1) follows, since $E_{D_{2}}$ is $\Theta_{D_{2}} \oplus \vartheta_{D_{2}}$ and $E_{D_{2}}^{\prime}$ is $\vartheta_{D_{2}} \oplus \Phi_{D_{2}} / \mathscr{G}_{D_{2}}^{2}$. Since $D_{2}^{2}=-1, E_{D_{2}}^{\prime} \cong \theta \oplus \vartheta(+1)$.

(5.1.2) and (5.1.4) follow from Remark 2.2. For (5.1.3), the standard quotient of $E_{D_{2}}^{\prime}$ is the canonical quotient, and $L_{1}$ is the quotient of $E_{P_{1}}$ which is glued to the canonical quotient of $\operatorname{Ker}\left(E_{P_{2}}, L_{2}\right)$.

Case $(1,0)$. If $\varphi$ has type $(1,0)$, we use the same surface $\mathcal{X}_{1}$ as in Case $(0,1)$. This time we have $\varphi: E_{P_{1}} \underset{\sim}{\rightarrow} E_{P_{2}}(-R)$. Letting $E^{(2)}$ be the pullback of $E$ to $\mathscr{X}_{1}$, we define

$$
E^{(3)}=\operatorname{Ker}\left(E^{(2)}, E_{D_{2}}^{(2)}\right)
$$

We see that

(5.2.1) $\quad E_{X}^{(3)}=E_{X}(-R)$.

(5.2.2) $E_{D}^{(3)}$ is $\Theta(1) \oplus \theta(1)$.

(5.2.3) $\quad E_{H_{2}}^{(3)}=E_{P_{2}}(-R)$.

Case $(1,1)$. We have a quotient $L_{2}$ of $E_{P_{2}}(-R)$ and

$$
\varphi: E_{P_{1}} \stackrel{\sim}{\rightarrow} \operatorname{Ker}\left(E_{P_{2}}(-R), L_{2}\right) \text {. }
$$

First, we proceed as in Case $(1,0)$. This time, however, we have

$$
\varphi: E_{H_{1}}^{(3)} \stackrel{\sim}{\rightarrow} \operatorname{Ker}\left(E_{H_{2}}^{(3)}, L_{2}\right) .
$$

So we blow up to $D_{2} \cap H_{2}$ to obtain a new surface $\mathcal{X}_{2}$. Thus $D_{3}$ is the new exceptional divisor, and $\mathrm{D}_{2}$ and $\mathrm{H}_{2}$ are the proper transforms of $\mathrm{D}_{2}$ and $\mathrm{H}_{2}$ from $\chi_{1}$. We let $E^{(4)}$ be the pullback of $E^{(3)}$ and let $E^{(5)}=\operatorname{Ker}\left(E^{(4)}, p^{*}\left(L_{2}\right)\right)$, where $p: \mathfrak{X}_{2} \rightarrow \mathfrak{X}_{1}$.

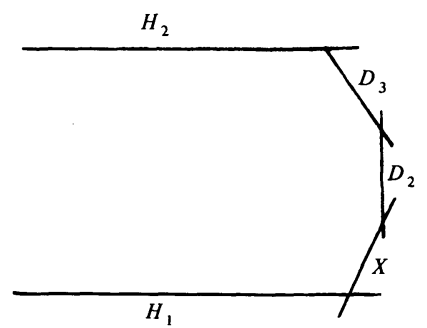

Now $E_{H_{2}}^{(5)}$ is isomorphic to $\operatorname{Ker}\left(E_{P_{2}}(-R), L_{2}\right)$ so $\varphi$ extends to an isomorphism of $E_{H_{1}}^{(5)}$ to $E_{H_{2}}^{(5)}$. Thus we can obtain our geometric realization by using $\varphi$ as glueing data for $E^{(5)}$. We claim:

(5.3.1) $E_{D_{2}}^{(5)}$ and $E_{D_{3}}^{(5)}$ are standard of degree 1. Further, their standard quotients do not coincide at $D_{2} \cap D_{3}$.

(5.3.2) $\quad E_{X}^{(5)}=E_{X \times R}(-R)$. 
Indeed, $E_{D_{3}}^{(4)}$ is $\vartheta_{D_{3}} \oplus \vartheta_{D_{3}}$ which we modify at a quotient $\vartheta_{D_{3}}$. Hence $E_{D_{3}}^{(5)}$ is $\vartheta_{D_{3}} \oplus \mathscr{G}_{D_{3}} / \mathscr{G}_{D_{3}}^{2}=\vartheta \oplus \vartheta(1)$. Further, the canonical quotient $E_{D_{3}}^{(5)}$ at $D_{2} \cap D_{3}$ is just the standard quotient of $E_{D_{3}}^{(5)}$. On the other hand, $E_{D_{2}}^{(5)}$ is $\operatorname{Ker}(\theta(1) \oplus$ $\left.\theta(1), L_{D_{2} \cap D_{3}}\right)$. Thus $E_{D_{2}}^{(5)}$ is $\theta \oplus \Theta(1)$, but the standard quotient does not coincide with the canonical quotient at $D_{2} \cap D_{3}$. Thus the standard quotients of $E_{D_{2}}^{(5)}$ and $E_{D_{3}}^{(5)}$ do not coincide.

Case $(-1,2)$. If $\varphi$ has type $(-1,2)$, then

$$
\varphi: E_{P_{1}} \stackrel{\sim}{\rightarrow} \operatorname{Ker}\left(E_{P_{2}}, L_{2}\right)(+R) .
$$

Let $L_{1}$ be the quotient of $E_{P_{1}}$ corresponding to the canonical quotient of $\operatorname{Ker}\left(E_{P_{2}}, L_{2}\right)(+R)$. Let $Q_{i}=L_{i} \otimes k_{R}$, where $k_{R}$ is $\theta_{S, R} / m$. We claim there is an isomorphism extending $\varphi$

$$
\psi: \operatorname{Ker}\left(E_{P_{1}}, Q_{1}\right) \stackrel{\sim}{\rightarrow} \operatorname{Ker}\left(E_{P_{2}}, Q_{2}\right) .
$$

Furthermore, $\psi$ does not identify the canonical quotients of the two sides. We call $Q_{1}$ and $Q_{2}$ the quotients induced by $\varphi$. Indeed, using the notation developed earlier in this section, $\varphi$ induces an isomorphism

$$
\varphi: N_{1}(-R) \oplus N_{2}(-R) \stackrel{\sim}{\rightarrow} M_{1} \oplus M_{2}(-2 R) .
$$

Now $\operatorname{Ker}\left(E_{P_{2}}, Q_{2}\right)$ is $M_{1} \oplus M_{2}(-R)$ and $\operatorname{Ker}\left(E_{P_{1}}, Q_{1}\right)$ is $N_{1}(-R) \oplus N_{2}$. Further, $\left(N_{2}\right)_{R}$ is the canonical quotient of $\operatorname{Ker}\left(E_{P_{1}}, Q_{1}\right)$ and $\left(M_{1}\right)_{R}$ is the canonical quotient of $\operatorname{Ker}\left(E_{P_{2}}, Q_{2}\right)$. Our claim follows.

Now form $X_{3}$ by blowing up $X_{1}$ at $P_{1}=X \cap H_{1}$ to obtain a new exceptional divisor $D_{1}$ (see the diagram). Let $E^{(6)}$ be the pullback of $E$ to $\mathscr{X}_{3}$ and let $E^{(7)}$ be the modification of $E^{(6)}$ at $p^{*}\left(Q_{1}\right) \oplus p^{*}\left(Q_{2}\right)$, where $p$ denotes the projection of $\mathscr{X}_{3}$ to $X \times S$.

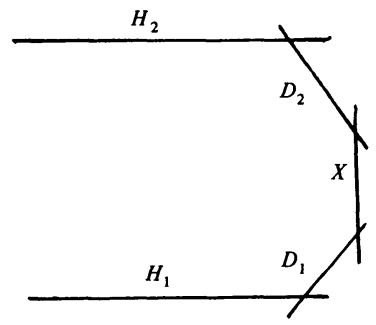

Then $\psi$ is an isomorphism of $E_{H_{1}}^{(7)}$ to $E_{H_{2}}^{(7)}$ and so we can use $\psi$ as descent data after identifying $H_{1}$ and $H_{2}$. We claim:

(5.4.1) $\quad E_{\mathrm{X}}^{(7)} \cong \operatorname{Ker}\left(E_{X}, Q_{1} \oplus Q_{2}\right)$.

(5.4.2) $E_{D_{i}}^{(7)}$ is standard to type 1 and the $\psi$ does not identify the standard quotients. 
These can be verified using the above techniques.

Now suppose $E$ is a family of stable bundles on $X$ of degree $2 \alpha+1$ over $S$ and let $\pi: X^{\prime} \rightarrow S$ be the geometric realization of $\varphi$. In Case $(0,1), X_{R}^{\prime}=X_{1}$ and $E_{R}^{\prime}$ is of type $\mathrm{II}_{1}$. Indeed, by Lemma 2.5 , there is no semistabilizing quotient of $E_{X}^{(2)}$ which is identified with the standard $D_{2}$ quotient of $E_{D_{2}}^{(2)}$.

If $\varphi$ is of type $(-1,0)$, then $X_{R}^{\prime}$ is $X_{1}$ and $E_{R}^{\prime}$ is of Type $\mathrm{II}_{2}$. If $\varphi$ is of type $(1,1)$, then $X_{R}^{\prime}$ is $X_{2}$, and $E_{R}^{\prime}$ is of Type III. Finally, if $\varphi$ is of type $(-1,2)$, Lemma 2.5(ii) shows that $X_{R}^{\prime}$ is $X_{2}$, and $E_{R}^{\prime}$ is of Type III unless there is an invertible quotient $L$ of degree $\alpha+1$ of $E_{X \times R}$ coinciding with $Q_{1}$ and $Q_{2}$ over $P_{1}$ and $P_{2}$.

6. We continue with the same notation as $\S 5$. We suppose that $\varphi$ has type $(-2,4)$. Let $L_{2}$ be the cokernel of the map from $E_{P_{1}}(-2 R)$ to $E_{P_{2}}$, and let $L_{1}$ be the quotient of $E_{P_{1}}$ corresponding to the canonical quotient of $\operatorname{Ker}\left(E_{P_{2}}, L_{2}\right)$. We suppose that there is an $X \times R$ quotient $L$ of $E$ which coincides with $L_{1}$ and $L_{2}$ at $R \times P_{1}$ and $R \times P_{2}$. Let $E^{\prime}=\operatorname{Ker}(E, L)$. Notice first that $\varphi$ has type $(-1,2)$ as a map of $E_{P_{1}}^{\prime}$ to $E_{P_{2}}^{\prime}$. Indeed, $\varphi$ induces an isomorphism

$$
\varphi: N_{1} \oplus N_{2} \rightarrow M_{1}(+2 R) \oplus M_{2}(-2 R) .
$$

Further, $L_{2}=M_{2} / M_{2}(-4 R)$ and $L_{1}=N_{1} / N_{1}(-4 R)$. Thus

$$
E_{P_{1}}^{\prime}=N_{1}(-R) \oplus N_{2}, \quad E_{P_{2}}^{\prime}=M_{1} \oplus M_{2}(-R) \text {. }
$$

Thus $E_{P_{1}}^{\prime}(-R)=N_{1}(-2 R) \oplus N_{2}(-R)$ and

$$
\operatorname{Coker}\left(E_{P_{1}}^{\prime}(-R), E_{P_{2}}^{\prime}\right)=M_{2}(-R) / \varphi\left(N_{2}(-R)\right) \text {, }
$$

which is isomorphic to $\theta_{R} / m^{2}$. So $\varphi$ has type $(-1,2)$ as a map from $E_{P_{1}}^{\prime}$ to $E_{P_{2}}^{\prime}$. Further, let $Q_{1}^{\prime}$ and $Q_{2}^{\prime}$ be the quotients of $E_{P_{1}}^{\prime}$ and $E_{P_{2}}^{\prime}$ induced by $\varphi$, and let $L^{\prime}$ be the canonical $X \times R$ quotient of $E^{\prime}=\operatorname{Ker}(E, L)$. Then $Q_{1}^{\prime}$ and $Q_{2}^{\prime}$ do not coincide with $L^{\prime}$ over $P_{1}$ and $P_{2}$. Indeed, $Q_{1}^{\prime}$ and $Q_{2}^{\prime}$ correspond to $N_{1}(-R)$ and $M_{2}(-R)$ at $P_{1}$ and $P_{2}$ and $L$ corresponds to $N_{2}$ and $M_{1}$ at $P_{1}$ and $P_{2}$. Let $S_{2}=\operatorname{Spec} \theta_{S, R} / m^{2}$.

Lemma 6.1. Suppose there is a $X \times R$ quotient $Q^{\prime}$ of $E^{\prime}$ so that $Q^{\prime}$ coincides with $Q_{1}^{\prime}$ and $Q_{2}^{\prime}$ over $P_{1}$ and $P_{2}$ and so that $E_{X \times R}^{\prime}$ is the direct sum of $Q^{\prime}$ and $L^{\prime}$. Then there is an $X \times S_{2}$ quotient of $E$ which coincides with $L_{1}$ and $L_{2}$ over $P_{1} \times S_{2}$ and $P_{2} \times S_{2}$ and with $L$ on $X \times R$.

Proof. Let $E^{\prime \prime}=\operatorname{Ker}\left(E^{\prime}, Q^{\prime}\right)$. We claim $E / E^{\prime \prime}$ is the desired quotient. First, we must show $E / E^{\prime \prime}$ is an $X \times S_{2}$ quotient. Since the problem is local, we may assume there is an isomorphism $\psi: E \rightarrow M^{\prime} \oplus M$ and that $M$ coincides with $L$ over $X \times R$. Then $E^{\prime}=M^{\prime} \oplus M(-D)$ where $D$ is the divisor $X \times R$. $M_{R}^{\prime}$ is the canonical quotient of $E^{\prime}$, so the map from $M(-D)$ to $Q^{\prime}$ is an isomorphism. Locally, we can modify $\psi$ so that the quotient $M(-D)$ of 
$M^{\prime} \oplus M(-D)$ coincides with $Q^{\prime}$. Then $E^{\prime \prime}=M^{\prime} \oplus M(-2 D)$, so $E / E^{\prime \prime}=$ $M / M(-2 D)$. Now at $P_{1}$ and $P_{2},\left(M_{2}(-R)\right)_{R}$ and $\left(N_{1}(-R)\right)_{R}$ coincide with the quotients induced by $\varphi$ on $E_{P_{1}}^{\prime}$ and $E_{P_{2}}^{\prime}$. Thus $E_{P_{1}}^{\prime \prime}=M_{1} \oplus M_{2}(-R)$ and $E_{P_{2}}^{\prime \prime}=N_{1}(-2 R) \oplus N_{2}$. Thus the $E_{P_{i}} / E_{P_{i}}^{\prime \prime}$ coincides with $L_{i}$ over $S_{2}$.

7. We wish to give a more global version of the construction of geometric realization of $\S 5$. Let $T$ be a smooth variety and $C_{1}, C_{2}, F_{1}$ and $F_{2}$ are smooth divisors meeting transversally (see the diagram). We assume there are no triple intersections, and that $C_{2}$ meets only $F_{2}, F_{2}$ meets only $C_{2}$ and $F_{1}$, and $C_{1}$ meets only $F_{1}$. Suppose that $E$ is a bundle on $X \times T$ and suppose we are given an isomoprhism

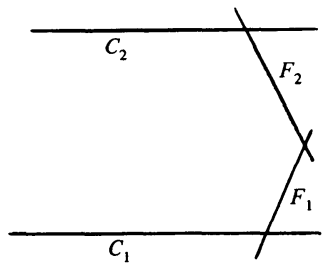

$$
\varphi: E_{P_{1}} \stackrel{\sim}{\rightarrow} \operatorname{Ker}\left(E_{P_{2}}, L_{2}\right)\left(C_{2}+F_{2}-C_{1}\right),
$$

where $L_{2}$ is an $F_{1}+F_{2}$ quotient of $E_{P_{2}}$. We let $L_{1}$ be the quotient of $E_{P_{1}}$ corresponding to the canonical $F_{1}+F_{2}$ quotient corresponding to the righthand side of (7.1).

Let $S$ be a smooth curve on $T$ and suppose $R \in S$. We suppose $S$ is transversal at $R$ to any $C_{i}$ or $F_{i}$ containing $R$. We let $\bar{E}$ be the restriction of $E$ to $S \times X$, and $\bar{\varphi}$ the induced rational map from $\bar{E}_{P_{1}}$ to $\bar{E}_{P_{2}}$. In the following list, the first column represents the position of $R$ and the second the type of $\bar{\varphi}$. Thus, if $R \in C_{2}-F_{2}, \bar{\varphi}$ has type $(-1,0)$ :

$$
\begin{array}{cc}
C_{2}-F_{2} & (-1,0) \\
C_{2} \cap F_{2} & (-2,1) \\
F_{2}-C_{2}-F_{1} & (-1,1) \\
F_{2} \cap F_{1} & (-1,2) \\
F_{1}-F_{2}-C_{1} & (0,1) \\
F_{1} \cap C_{1} & (1,1) \\
F_{1}-D_{1} & (1,0)
\end{array}
$$

Our aim in this section is to construct a family of curves $q: Y \rightarrow T$ and a bundle $F$ on $Y$ so that for any $S$ as above, the geometric realization of $\bar{\varphi}$ is just the pullback of $Y$ and $F$ to $S$. First, on $T \times X$ blow up $C_{1} \times P_{1}$ and $C_{2} \times P_{2}$ to obtain a variety $W_{1}$ mapping to $T \times X$. Let $H_{1}$ and $H_{2}$ be the proper 
transforms of $T \times P_{1}$ and $T \times P_{2}$, and let $V_{1}$ and $V_{2}$ be the exceptional divisors. Then $H_{i}$ maps isomorphically to $T$, and $V_{i} \cap H_{i}$ maps to $C_{i}$. Now denoting the pullback of $E$ by $E$ again,

$$
\varphi: E_{H_{1}} \rightarrow \operatorname{Ker}\left(E_{H_{2}}, L_{2}\right)\left(C_{2}+F_{2}-C_{1}\right) .
$$

Now on $W_{1}$, let

$$
E^{\prime}=\operatorname{Ker}\left(E, E_{V_{1}} \oplus E_{V_{2}}\right) .
$$

Then $\varphi$ induces an isomorphism

$$
\varphi: E_{H_{1}}^{\prime} \rightarrow \operatorname{Ker}\left(E_{H_{2}}^{\prime}, L_{2}^{\prime}\right)\left(+F_{2}\right),
$$

where $L_{2}^{\prime}$ is the $F_{1}+F_{2}$ quotient of $E_{H_{2}}^{\prime}$ given by $L_{2}^{\prime}=L_{2}\left(-C_{2}+C_{1}\right)$. Now let $L_{1}^{\prime}$ be the $F_{1}+F_{2}$ quotient of $E_{H_{1}}^{\prime}$ corresponding to the canonical quotient of $\operatorname{Ker}\left(E_{H_{2}}^{\prime}, L_{2}^{\prime}\right)$. Then $\varphi$ induces an isomorphism

$$
\varphi_{2}: \operatorname{Ker}\left(E_{H_{1}}^{\prime},\left(L_{1}^{\prime}\right)_{F_{2}}\right) \stackrel{\sim}{\rightarrow} \operatorname{Ker}\left(E_{H_{2}}^{\prime},\left(L_{2}^{\prime}\right)_{F_{1}}\right)
$$

Indeed, it is easy to check that $\varphi_{2}$ is an isomorphism over $F_{1}-F_{2}$ and over $F_{2}-F_{1} . \varphi_{2}$ is therefore an isomorphism since $\wedge^{2} \varphi_{2}$ does not vanish except possibly on $F_{1} \cap F_{2}$ and hence does not vanish on $F_{1} \cap F_{2}$.

Next in $H_{1}$, we have the smooth variety $F_{2}^{\prime}$ corresponding to $F_{2}$ under the projection of $H_{1}$ to $T$. We can similarly define $F_{1}^{\prime}$. Now blow up $F_{2}^{\prime}$ and $F_{1}^{\prime}$ in $W_{1}$ to obtain $W_{2}$. Let $H_{i}$ denote the proper transform of $H_{i}$ in $W_{2}$ and let $G_{1}$ and $G_{2}$ be the new exceptional divisors. We will let $E^{\prime}$ denote the pullback of $E^{\prime}$ to $W_{2}$ and $M_{1}$ and $M_{2}$ be the pullbacks $\left(L_{2}^{\prime}\right)_{F_{1}}$ and $\left(L_{1}^{\prime}\right)_{F_{2}}$. Then $M_{1}$ and $M_{2}$ are $G_{1}$ and $G_{2}$ quotients of $E^{\prime}$ on $W_{2}$. Let

$$
E^{\prime \prime}=\operatorname{Ker}\left(E^{\prime}, M_{1} \oplus M_{2}\right) \text {. }
$$

Then $\varphi$ induces an isomorphism of $E_{H_{1}}^{\prime \prime}$ with $E_{H_{2}}^{\prime \prime}$. We glue $H_{1}$ to $H_{2}$ in $W_{2}$ to form our family of curves $q: Y \rightarrow T$ and use $\varphi$ as descent data for $E^{\prime \prime}$. One checks that if $S \subseteq T$ is a curve as above, then the geometric realization of $\bar{\varphi}$ is just the pullback of $Y$ and $F$.

8. Let $S_{0}$ be the moduli space of stable bundles of degree $2 \alpha+1$ on $X$ and let $E$ be the Poincaré bundle on $X \times S_{0}$. Our object is to construct the normalization of $\mho_{P}$ from $S_{0}$ and $E$, where $\mathscr{W}$ is the variety introduced at the end of $\S 4$. We first consider

$$
S_{1}=\mathbf{P}\left(\operatorname{Hom}\left(E_{P_{2}}, E_{P_{1}}\right)\right)
$$


as a projective bundle over $S_{0}$. Let $M_{1}$ be the tautological bundle of $\pi_{1}$ : $S_{1} \rightarrow S_{0}$. We will denote the pullback of $E$ to $S_{1} \times X$ by $E^{(1)}$. Now on $S_{0}$, there is the usual exact sequence

$$
\operatorname{Hom}\left(E_{P_{2}}^{(1)}, E_{P_{1}}^{(1)}\right) \rightarrow M_{1} \rightarrow 0
$$

and thus a map

$$
\varphi_{1}: E_{P_{1}}^{(1)} \rightarrow E_{P_{2}}^{(1)} \otimes M_{1} \text {. }
$$

One checks locally that $\varphi_{1}$ is nowhere zero and that $\wedge^{2} \varphi_{1}$ vanishes simply on a smooth divisor $D$ which is a bundle of quadrics over $S_{0}$.

$$
\vartheta(D) \cong M_{1}^{2} \otimes \wedge^{2} E_{P_{2}}^{(1)} \otimes\left(\wedge^{2} E_{P_{1}}^{(1)}\right)^{-1} \text {. }
$$

There is a $D$ quotient $L_{2}^{(1)}$ of $E_{P_{2}}^{(1)}$ so that

$$
\varphi_{1}: E_{P_{1}}^{(1)} \stackrel{\sim}{\rightarrow} \operatorname{Ker}\left(E_{P_{2}}^{(1)} \otimes M_{1}, L_{2}^{(1)} \otimes M_{1}\right) .
$$

We let $L_{1}^{(1)}$ be the quotient of $E_{P_{1}}^{(1)}$ corresponding to the canonical quotient of the left-hand side of (8.1.2).

Consider

$$
S_{2}=\mathbf{P}\left(\theta \oplus M_{1}\right)
$$

as a projective bundle over $S_{1}$.

Let $M_{2}$ be the tautological line bundle for $\pi_{2}: S_{2} \rightarrow S_{1}$. Let $E^{(2)}$ be the pullback of $E^{(1)}$ to $X \times S_{2}$, and $M_{1}^{(2)}$ be the pullback of $M_{1}$. We have the exact sequence on $S_{2}$

$$
\vartheta \oplus M_{1}^{(2)} \rightarrow M_{2} \rightarrow 0 .
$$

There are divisors $H_{1}$ and $H_{2}$ which are sections of $\pi_{2}$ so that the map from $\theta$ to $M_{2}$ vanishes on $H_{2}$ and the map from $M_{1}^{(2)}$ vanishes on $H_{1}$. So we have isomorphisms $\stackrel{\vartheta}{\sim} \underset{\rightarrow}{\sim} M_{2}\left(-H_{2}\right)$ and $M_{1}^{(2)} \stackrel{\sim}{\rightarrow} M_{2}\left(-H_{1}\right)$. We obtain an isomorphism

$$
\psi: \vartheta\left(H_{2}-H_{1}\right) \stackrel{\sim}{\rightarrow} M_{1}^{(2)} .
$$

Thus we have a morphism

$$
\varphi_{2}: E_{P_{1}}^{(2)} \rightarrow E_{P_{2}}^{(2)}\left(H_{2}-H_{1}\right) .
$$

We have the following schematic picture of $S_{2}$ where $D^{(2)}=\pi_{2}^{-1}(D)$ :

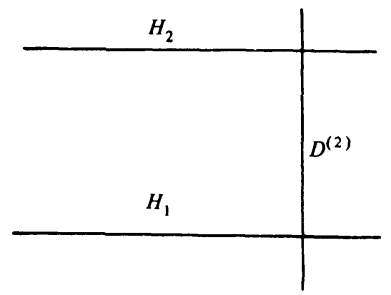


Consider $U=S_{2}-H_{1}-H_{2}-D^{(2)}$, and let $V_{0} \subseteq W_{P}$ be the open set of bundles of Type $\mathrm{I}_{s}$. Note $\varphi_{2}$ is an isomorphism over $U$ and we can use $\varphi_{2}$ as descent data to produce a bundle $E^{\prime}$ on $X_{0} \times U . E^{\prime}$ is a family of bundle of Type $\mathrm{I}_{s}$ on $X_{0}$ and so we get a map $\psi_{0}$ of $U$ to $V_{0}$. We claim $\psi_{0}$ is an isomorphism. Suppose $T$ is a $k$ scheme and $E^{\prime \prime}$ is a family of bundles on $T \times X_{0}$ of Type $\mathrm{I}_{s}$. By pullback, we obtain a family of stable bundles $\tilde{E}$ on $T \times X$ together with an isomorphism $\tilde{\varphi}: \tilde{E}_{P_{1}} \rightarrow \tilde{E}_{P_{2}}$. There is a morphism $F_{0}$ of $T$ to $S_{0}$ so that $\tilde{E}$ is the pullback of $E$ up to tensoring by line bundles on $T$. From the universal property of $\mathbf{P}\left(\operatorname{Hom}\left(E_{P_{2}}, E_{P_{1}}\right)\right)$, there is lifting $P_{1}$ of $F_{0}$ to $S_{1}$ and an isomorphism $h: F_{1}^{*}\left(M_{1}\right) \rightarrow \theta$ so that $\tilde{\varphi}$ is the pullback of $\varphi_{1}$ when we use $h$ to identify $\theta$ with $F_{1}^{*}\left(M_{1}\right)$. But $h$ determines the lifting $F_{2}$ of $F_{1}$ to $S_{2}=\mathbf{P}\left(\theta \oplus M_{1}\right)$ so that $h$ is given as $\rho_{1}^{-1} \rho_{2}$, where $\rho_{2}$ is the map of $\theta$ to $F_{2}^{*}\left(M_{2}\right)$ and $\rho_{1}$ is the isomorphism of $F_{1}^{*}\left(M_{1}\right) \rightarrow F_{2}^{*}\left(M_{2}\right)$. Thus $\tilde{\varphi}$ is just the pullback of $\varphi_{2}$. Hence, we obtain a map of $T$ to $U$. Let $V \subseteq \mathscr{Y}$ be the open set consisting of curves of Type $\mathrm{I}_{s}$. Then we have a map of $V$ to $U$ which is $\operatorname{SL}(W)$ invariant. Thus, we get a map $\psi_{1}$ of $V_{0}$ to $U$. We leave it to the reader to check that $\psi_{0}$ and $\psi_{1}$ are inverse maps.

It is impossible to extend $\psi_{0}$ to a map of $S_{2}$ to $\mho_{P}$. So we must blow up $S_{2}$. First, blow up $\mathrm{H}_{2} \cap D^{(2)}$ to obtain $S_{3}$ :

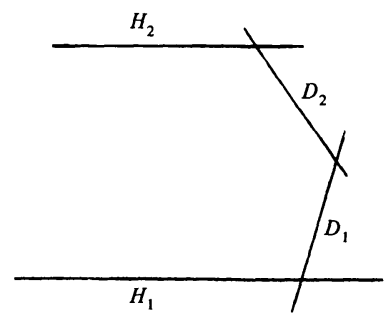

Here $H_{2}$ is the proper transform of $H_{2}, D_{2}$ is the new exceptional divisor, and $D_{1}$ is the proper transform of $D^{(2)}$. Thus, the total transform of $D^{(2)}$ is $D_{1}+D_{2}$ and the total transform of $\mathrm{H}_{2}$ is $\mathrm{H}_{2}+D_{2}$. Let $E^{(3)}$ be the pullback of $E^{(2)}$ to $S_{3}$ and let $L_{2}^{(3)}$ be the pullback of $L_{2}^{(1)} \cdot L_{2}^{(3)}$ is a $D_{1}+D_{2}$ quotient of $E_{P_{2}}^{(3)}$ and we have an isomorphism

$$
\varphi_{3}: E_{P_{1}}^{(3)} \rightarrow \operatorname{Ker}\left(E_{P_{2}}^{(3)}, L_{2}^{(3)}\right)\left[H_{2}+D_{2}-H_{1}\right]
$$

extending $\varphi_{2}$ on $U$. Thus, we are in the situation of $\S 7$. Let $L_{1}^{(3)}$ be the $D_{1}+D_{2}$ quotient of $E_{P_{1}}^{(3)}$ corresponding to the canonical quotient of the right-hand side of (8.1.3). Let $Z^{\prime}$ be the subset of $D_{1} \cap D_{2}$ consisting of points $s \in S_{3}$ so that $E_{3}$ has a quotient of degree $\alpha+1$ which coincides with $L_{i}^{(3)}$ in $\left(E_{s}\right)_{P_{i}}$. From the 
discussion at the end of $\$ \S 5$ and 7 , one sees that the family of curves and bundle obtained as the geometric realization of $\varphi_{3}$ is stable except over $Z^{\prime}$.

9. Our purpose in this section is to understand $Z^{\prime}$. Let $J_{d}$ be the Picard variety of line bundles of degree $d$ on $X$ and let $\mathcal{L}_{d}$ be the Poincare bundle on $X \times J_{d}$. We will denote the pullbacks of $\mathcal{L}_{\alpha}$ and $\mathcal{L}_{\alpha+1}$ to $X \times J_{\alpha} \times J_{\alpha+1}$ by $\varrho_{\alpha}$ and $\mathcal{L}_{\alpha+1}$. Let $\pi_{1}$ be the projection of $X \times J_{\alpha} \times J_{\alpha+1}$ onto $J_{\alpha} \times J_{\alpha+1}$. First consider the sheaf on $J_{\alpha} \times J_{\alpha+1}$

$$
\mathscr{F}_{1}=R^{1} \pi_{1^{*}}\left(\mathfrak{L}_{\alpha+1}^{-1} \otimes \varrho_{\alpha}\right) \text {. }
$$

First, note that $\mathscr{F}_{1}$ is locally free of rank $g-1$ since if $L$ is a line bundle of degree -1 on $X, h^{1}(L)=g-1$. (The genus of $X$ is $g-1$.) Let $Z=\mathbf{P}\left(\mathscr{F}_{1}^{-1}\right)$. We will construct an isomorphism of $Z$ with $Z^{\prime}$. Note $\operatorname{dim} Z=3 g-4$.

Again denoting the pullbacks of $\mathcal{L}_{\alpha+1}$ and $\mathcal{L}_{\alpha}$ to $Z \times X$ by $\mathcal{L}_{\alpha}$ and $\mathcal{L}_{\alpha+1}$ and letting $\pi_{2}$ be the projection of $Z \times X$ to $Z$, we have a canonical section $s$ of $\Re \otimes R^{1} \pi_{2^{*}}\left(\mathfrak{L}_{\alpha+1}^{-1} \otimes \mathcal{L}_{\alpha}\right)$, where $\Re$ is the tautological bundle on $Z$ over $J_{\alpha} \times$ $J_{\alpha+1}$.

Now $s$ defines an extension

$$
0 \rightarrow \mathfrak{L}_{\alpha} \otimes \Re \rightarrow \mathcal{E} \rightarrow \mathfrak{L}_{\alpha+1} \rightarrow 0
$$

For each $z \in Z$, the corresponding extension

$$
0 \rightarrow\left(\mathscr{L}_{\alpha} \otimes \Re\right)_{z} \rightarrow \mathcal{E}_{z} \rightarrow\left(\varrho_{\alpha+1}\right)_{z} \rightarrow 0
$$

is nontrivial. We claim $\mathcal{E}_{z}$ is stable. Indeed, if $Q_{z}$ is a destabilizing quotient of $\mathcal{E}_{z}$, the induced map $f$ from $\left(\mathfrak{L}_{\alpha} \otimes \mathfrak{K}\right)_{z}$ to $Q_{z}$ is nontrivial. But if $f$ is zero at any point, $\operatorname{deg} Q_{z}>\alpha$ so $Q_{z}$ is not destabilizing. But the extension is trivial if $f$ is an isomorphism.

Let $T$ be a $k$-scheme. Let $G(T)$ be the set of extensions (modulo equivalence)

$$
0 \rightarrow L_{\alpha} \rightarrow F \rightarrow L_{\alpha+1} \rightarrow 0
$$

on $X \times T$, where $L_{\alpha}$ and $L_{\alpha+1}$ have degree $\alpha$ and $\alpha+1$ on $X \times\{t\}$ and where $F$ is a family of stable bundles on $X$ over $T$. Using the universal properties of the Picard group and $\mathbf{P}\left(\mathscr{F}_{1}^{-1}\right)$, one sees that $Z$ represents $G$.

We wish to define another functor $G^{\prime}$. An element of $G^{\prime}(T)$ will consist of a family $F$ of stable bundles on $X \times T$ together with $T \times P_{i}$ quotients $\mathfrak{L}_{i}$ of $F$ (modulo equivalence). Recall the divisor $D \subseteq S_{1}$ from $\S 8$ and the $D$ quotients $L_{i}^{(1)}$ of $E_{P_{i}}^{(1)}$. We claim $D$ represents the functor $G^{\prime}$. Indeed, given $\left(F, \varrho_{1}, \varrho_{2}\right) \in$ $G^{\prime}(T)$, we can locally on $T$ find a $\varphi: F_{P_{1}} \rightarrow F_{P_{2}}$ so that coker $\varphi=\mathcal{L}_{2}$ and $F_{P_{1}} / \operatorname{Ker} \varphi=\mathcal{L}_{1}$. Further, $\varphi$ is uniquely determined up to a unit on $T$. Using the universal properties of $S$ and $S_{1}$, one obtains a well-defined map from $T$ to $D$ so that $\left(F, \varrho_{1}, \varrho_{2}\right)$ is the pullback of $\left(E^{(1)}, L_{1}^{(1)}, L_{2}^{(1)}\right)$ modulo isomorphism. 
There is a map

$$
\Psi(T): G(T) \rightarrow G^{\prime}(T)
$$

obtained by letting $\varrho_{i}=\left(L_{\alpha+1}\right)_{P_{i}}$. We claim $\Psi$ is injective for any $T$. Indeed, suppose $Q$ is a quotient of degree $\alpha+1$ of $F$ in (9.1.2) which agrees with $L_{\alpha+1}$ at $P_{i} \times T$. Consider the map $f$ of $L_{\alpha}$ to $Q$. This is actually a map of $L_{\alpha}$ to $Q\left(-P_{1}-P_{2}\right)$. Since $\operatorname{deg} L_{\alpha}>\operatorname{deg} Q\left(-P_{1}-P_{2}\right), f$ is zero. Thus $\Psi(T)$ is injective.

Proposition 9.2. The induced map of $Z$ to $D$ is an embedding.

Amplification 9.3. Let $T=\operatorname{Spec} k[\varepsilon] /\left(\varepsilon^{2}\right)$ and let $R$ be the closed point of $T$. Let $\left(F, \mathcal{L}_{1}, \mathcal{L}_{2}\right) \in G^{\prime}(T)$. Then the induced map of $T$ to $D$ factors through $Z$ if and only if there is a $T$ quotient $L_{\alpha+1}$ of $F$ degree $\alpha+1$ coinciding with $\varrho_{1}$ and $L_{2}$.

10. We retain the notation of $\S 8$. Using the map of $Z$ to $D$ and the isomorphism of $D_{1} \cap D_{2}$ with $D$, we can identify $Z$ with $Z^{\prime}$. We form a new variety $S_{4}$ by blowing up $Z \subseteq S_{3}$. We will call the new exceptional divisor $\tilde{Z}$ and denote the proper transforms of the $D_{i}$ by $D_{i}$.

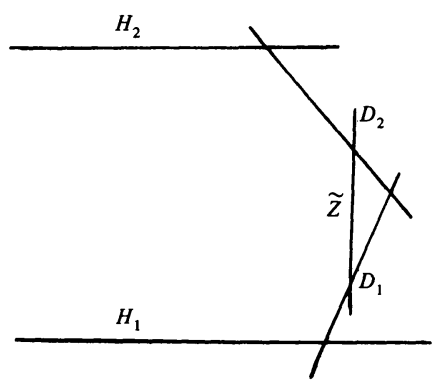

Our main result of this section is

Proposition 10.1. $\psi_{0}$ extends to a morphism of $S_{4}$ to $\mho_{P}$.

Let $E^{(4)}$ be the pullback of $E^{(3)}$ and $L_{2}^{(4)}$ the pullback of $L_{2}^{(3)}$. Then $L_{2}^{(4)}$ is a $D_{1}+D_{2}+2 \tilde{Z}$ quotient of $E_{P_{2}}^{(4)}$ and there is an isomorphism

$$
\varphi_{4}: E_{P_{1}}^{(4)} \rightarrow \operatorname{Ker}\left(E_{P_{2}}^{(4)}, L_{2}^{(4)}\right)\left[H_{2}+D_{2}+\tilde{Z}-H_{1}\right] .
$$

$L_{1}^{(4)}$ will denote the quotient of $E_{P_{1}}^{(4)}$ corresponding to the canonical quotient of the right side of (10.1.1). Now let $\mathcal{L}_{\alpha+1}^{\prime}$ be the invertible quotient of $E_{Z}^{(3)}$ of degree $\alpha+1$ which coincides with $L_{i}^{(3)}$ over $P_{i}$. Let $\tilde{\mathcal{L}}_{\alpha+1}$ be the pullback of $\mathcal{L}_{\alpha+1}$ to $\tilde{Z}$. Further, let

$$
E^{\prime}=\operatorname{Ker}\left(E^{(4)}, \tilde{\mathfrak{e}}_{\alpha+1}\right)
$$


Now let $L_{i}^{\prime}=L_{i}^{(4)} \otimes \vartheta_{D_{1}+D_{2}}(-\tilde{Z})$. Note that $L_{i}^{\prime}$ is a $D_{1}+D_{2}$ quotient of $E_{P_{i}}^{\prime}$. We claim there is an isomorphism

$$
\varphi^{\prime}: E_{P_{1}}^{\prime} \rightarrow \operatorname{Ker}\left(E_{P_{2}}^{\prime}, L_{2}^{\prime}\right)\left(H_{2}+D_{2}-H_{1}\right)
$$

which coincides with $\varphi_{4}$ away from $\tilde{Z}$ and so that $L_{1}^{\prime}$ coincides with the canonical quotient of $\operatorname{Ker}\left(E_{P_{2}}^{\prime}, L_{2}^{\prime}\right)$ under $\varphi^{\prime}$. Our main object in this section is to show that the geometric realization of $\varphi^{\prime}$ is stable over all points of $S_{4}$.

To show that the isomorphism $\varphi^{\prime}$ exists, it suffices to work locally around a point of $\tilde{Z}$. Thus we may assume that

$$
E_{P_{1}}^{(4)}=\varrho_{1} \oplus \Re_{1}, \quad E_{P_{2}}^{(4)}=\varrho_{2} \oplus \Re_{2},
$$

where $\mathscr{L}_{i}$ coincides with $L_{i}^{(4)}$ over $D_{1}+D_{2}+2 \tilde{Z}$ and so that $\varphi_{4}=\varphi_{1} \oplus \varphi_{2}$ where $\varphi_{1}$ is an isomorphism of $\mathcal{L}_{1}$ with $\Re_{2}\left(D_{2}+\tilde{Z}\right)$ and $\varphi_{2}$ is an isomorphism of $\Re_{1}$ with $\mathcal{L}_{2}\left(-D_{1}-\tilde{Z}\right)$. Now $E_{P_{1}}^{\prime}=\mathscr{L}_{1}(\tilde{Z}) \oplus \Re_{1}$ and $E_{P_{2}}^{\prime}=\varrho_{2}(-\tilde{Z}) \oplus \Re_{2}$ and hence

$$
\begin{aligned}
\operatorname{Ker}\left(E_{P_{2}}^{\prime}, L_{2}^{\prime}\right)\left(D_{2}\right) & =\left(\varrho_{2}\left(-\tilde{Z}-D_{1}-D_{2}\right) \oplus \Re_{2}\right)\left(D_{2}\right) \\
& =\varrho_{2}\left(-\tilde{Z}-D_{1}\right) \oplus \Re_{2}\left(D_{2}\right) .
\end{aligned}
$$

Thus $\varphi^{\prime}$ exists, since $E_{P_{1}}^{\prime}=\varrho_{1}(-\tilde{Z}) \oplus \Re_{1}$.

Let $Q$ be the canonical $\tilde{Z}$ quotient of $E^{\prime}$ from (10.1.2). $Q_{P_{1}}$ and $Q_{P_{2}}$ are just $\left(\Re_{P_{1}}\right)_{Z}$ and $\left(\Re_{P_{2}}\right)_{Z}$. So $Q_{P_{2}}$ is actually a quotient of $\operatorname{Ker}\left(E_{P}^{\prime}, L_{2}^{\prime}\right)$. Further, $Q_{P}$ is never glued to $Q_{P_{2}}\left(D_{2}\right)$ under $\varphi^{\prime}$ and $Q_{P_{i}}$ never coincides with $L_{i}^{\prime}$ as a quotient of $E_{P_{i}}^{\prime}$. Let $N_{1}$ be the quotient of $\left(E_{P_{1}}^{\prime}\right) \tilde{z}$ which corresponds to $Q_{P_{2}}\left(D_{2}\right)$ under $\varphi^{\prime} . Q_{P_{2}}$ is a quotient of $\operatorname{Ker}\left(E_{P_{2}}^{\prime}, L_{2}^{\prime}\right)$ which coincides with the canonical quotient of $\operatorname{Ker}\left(E_{P_{2}}^{\prime}, L_{2}^{\prime}\right)$ over $\left(D_{1}+D_{2}\right) \cap \tilde{Z}$. Thus $N_{1}$ corresponds to $L_{1}^{\prime}$ over $\left(D_{1}+D_{2}\right) \cap \tilde{Z}$.

Since $L_{1}^{\prime}$ corresponds to the canonical quotient of $\operatorname{Ker}\left(E_{P_{2}}^{\prime}, L_{2}^{\prime}\right)\left(D_{2}\right)$, one sees there is an isomorphism near $\tilde{Z}$

$$
\psi^{\prime}: E_{P_{2}}^{\prime} \rightarrow \operatorname{Ker}\left(E_{P_{1}}^{\prime}, L_{1}^{\prime}\right)\left(+D_{1}\right)
$$

which is the inverse of $\varphi^{\prime}$ except over $D_{1}+D_{2}$. One defines $N_{2}$ analogously to $N_{1}$.

Now we have an exact sequence

$$
0 \rightarrow M \rightarrow E_{\tilde{Z} \times X}^{\prime} \rightarrow Q \rightarrow 0,
$$

where $M$ is a line bundle on $\tilde{Z} \times X$. Since the map $\varphi^{\prime}$ never identifies $Q_{P_{1}}$ with $Q_{P_{2}}\left(+D_{1}\right)$, the map from $M_{P_{1}}$ to $Q_{P_{2}}\left(+D_{1}\right)$ is an isomorphism. So we get a map $\theta_{1} \in \operatorname{Hom}\left(Q_{P_{2}}, M_{P_{1}}\right)$ which vanishes on $D_{1}$. Similarly, using $\psi^{\prime}$ one defines $\theta_{2} \in \operatorname{Hom}\left(Q_{P_{1}}, M_{P_{2}}\right)$ which vanishes on $D_{2}$. 
Finally, let

$$
E^{\prime \prime}=\operatorname{Ker}\left(E_{\tilde{Z} \times X}^{\prime}, N_{1} \oplus N_{2}\right) .
$$

Note that $Q$ is a quotient of $E^{\prime \prime}$ and that the kernel of $E^{\prime \prime} \rightarrow Q$ is just $M\left(-P_{1}-P_{2}\right)$. Thus we obtain an extension on $\tilde{Z} \times X$

$$
0 \rightarrow M\left(-P_{1}-P_{2}\right) \rightarrow E^{\prime \prime} \rightarrow Q \rightarrow 0
$$

and so a section

$$
\theta_{3} \in H^{0}\left(\tilde{Z}, R^{1} \pi_{*}\left(M \otimes Q^{-1}\left(-P_{1}-P_{2}\right)\right)\right),
$$

where $\pi$ is the projection of $\tilde{Z} \times X$ to $\tilde{Z}$.

Now suppose $R \in \tilde{Z}$. We wish to examine the stability of the geometric realization $\left(X_{R}, F_{R}\right)$ of $\varphi^{\prime}$ over $R$. First, suppose that $R \notin D_{1}+D_{2}$. Then $\varphi_{R}^{\prime}$ : $\left(E_{P}^{\prime}\right)_{R} \rightarrow\left(E_{P_{2}}^{\prime}\right)_{R}$ is an isomorphism and we have an exact sequence

$$
0 \rightarrow M_{R} \rightarrow E_{R}^{\prime} \rightarrow Q_{R} \rightarrow 0
$$

on $X \times R=X$. Now we have observed that $Q_{P_{1}}$ and $Q_{P_{2}}$ are not identified under $\varphi_{R}^{\prime}$. Thus $F_{R}$ on $X_{0}$ is of Type $\mathrm{I}_{u}$, since $\operatorname{deg} Q_{R}=\alpha+1$ and $\operatorname{deg} M_{R}=\alpha$. Suppose $P$ is another point of $\tilde{Z}-D_{1}-D_{2}$.

Lemma 10.2. Suppose $M_{P}$ and $M_{R}$ are isomorphic and that $Q_{P}$ and $Q_{R}$ are isomorphic. Suppose that under these isomorphisms we have $\theta_{i}(R)=\lambda \theta_{i}(P)$ for $i=1,2,3$ and $\lambda \in k^{*}$. Then $F_{R}$ is isomorphic to $F_{P}$.

Proof. We may first assume that $\lambda=1$ by multiplying the isomorphism of $M_{P}$ with $M_{R}$ by a suitable constant. Since $\theta_{3}(R)=\theta_{3}(P)$, we can find an isomorphism $\psi$ of the extensions:

$$
\begin{aligned}
& 0 \rightarrow M_{R}\left(-P_{1}-P_{2}\right) \rightarrow E_{R}^{\prime \prime} \rightarrow Q_{R} \rightarrow 0 \\
& 0 \rightarrow M_{P}\left(-P_{1}-P_{2}\right) \rightarrow E_{P}^{\prime \prime} \rightarrow Q_{P} \rightarrow 0 .
\end{aligned}
$$

Now

$$
E_{R}^{\prime}=\operatorname{Ker}\left(E_{R}^{\prime \prime}, Q_{P_{1}} \oplus Q_{P_{2}}\right)\left(P_{1}+P_{2}\right)
$$

Thus there is an isomorphism of the extension (10.1.3) with the analogous extension over $P$ so that $\left(N_{i}\right)_{R}$ corresponds to $\left(N_{i}\right)_{P}$. But $\left(N_{1}\right)_{R}$ is just the quotient of $\left(E_{P_{1}}^{\prime}\right)_{R}$ corresponding to $\left(Q_{P_{2}}\right)_{R}$. Further, $\theta_{1}(R)$ just gives the map from $\left(M_{P_{1}}\right)_{R}$ to $\left(Q_{P_{2}}\right)_{R}$. Using the corresponding statements for $\left(N_{2}\right)_{R}$ and for $P$, our lemma follows from the following observation: Suppose $V_{1}$ and $V_{2}$ are two two-dimensional vector spaces given as extensions

$$
0 \rightarrow U_{i} \rightarrow V_{i} \rightarrow W_{i} \rightarrow 0,
$$

and that $\psi$ and $\psi^{\prime}$ are isomorphisms of $V_{1}$ with $V_{2}$. Suppose $\psi\left(U_{1}\right)=\psi^{\prime}\left(U_{1}\right)$, $\psi^{-1}\left(U_{2}\right)=\left(\psi^{\prime}\right)^{-1}\left(U_{2}\right)$ and the induced maps of $U_{1}$ to $W_{2}$ and $U_{2}$ to $W_{1}$ are equal. Then $\psi=\psi^{\prime}$. 
Next suppose $R \in\left(\tilde{Z} \cap D_{1}\right)-D_{2}$. The quotient $\left(L_{2}^{\prime}\right)_{R}$ does not coincide with $\left(Q_{P_{2}}\right)_{R}$. Let $E^{\prime \prime}=\operatorname{Ker}\left(E_{R}^{\prime}, L_{R}^{\prime}\right)$. Thus we have an exact sequence

$$
0 \rightarrow M_{R}\left(-P_{2}\right) \rightarrow E^{\prime \prime} \rightarrow Q_{R} \rightarrow 0 \text {. }
$$

Thus $E^{\prime \prime}$ is semistable and $\left(Q_{R}\right)_{P_{2}}$ is the canonical $P_{2}$ quotient of $E^{\prime \prime}$. On the other hand, $\left(N_{1}\right)_{R} \neq\left(Q_{R}\right)_{P_{1}}$. From (5.1.1)-(5.1.4), we see that $F_{R}$ is on $X_{1}$, $\left(F_{R}\right)_{X}=E^{\prime \prime},\left(Q_{R}\right)_{P_{2}}$ is glued to the standard quotient of $\left(F_{R}\right)_{R_{1}}=\vartheta \oplus \theta(1)$ and $\left(Q_{R}\right)_{P_{1}}$ is not glued to the standard quotient of $\left(F_{R}\right)_{R_{1}}$. Thus $F_{R}$ is of Type $\mathrm{II}_{1}$.

Finally, suppose $R \in \tilde{Z} \cap D_{1} \cap D_{2}$. Let $C$ be a curve passing through $R$ transversal to $\tilde{Z}, D_{1}$ and $D_{2}$. We claim we cannot write

$$
E_{R}^{\prime}=M_{R} \oplus Q_{R}
$$

where the quotient $M_{R}$ coincides with $\left(L_{i}^{\prime}\right)_{R}$ over $P_{i}$. Now $\varphi_{C}^{\prime}$ has type $(-2,4)$ as a map from $\left(E_{C}\right)_{P_{1}}$ to $\left(E_{C}\right)_{P_{2}}$. If $(10.2 .1)$ holds, Lemma 6.1 shows there is a $X \times C_{2}$ quotient of $E_{C}^{(4)}$ which coincides with $\tilde{\mathfrak{e}}_{\alpha+1}$ on $X \times R$ and which coincides with $L_{i}^{(4)}$ over $P_{i} \times C_{2}$. But from Amplification 9.3, we see that the map from $C_{2}$ to $S_{1}$ factors through $Z$, i.e., the image of $C$ in $S_{3}$ is tangent to $Z$. But $C$ meets $\tilde{Z}$ transversally, and hence the image of $C$ meets $Z$ transversally. Thus our claim is established.

$F_{R}$ is a bundle on $X_{2}$ and $\left(F_{R}\right)_{X}=\operatorname{Ker}\left(E_{R}^{\prime},\left(L_{1}^{\prime} \oplus L_{2}^{\prime}\right)_{R}\right)$. The $L_{i}^{\prime}$ coincide with $N_{i}$ over $R$, and the $N_{i}$ do not coincide with the $Q_{i}$. Lemma 2.6(ii) shows that $\left(F_{R}\right)_{X}$ is stable, and hence $F_{R}$ is of Type III. Thus we have completed the proof of Proposition 10.1.

Lemma 10.3. For any point $R \in \tilde{Z}$, at least one of the $\theta_{i}$ 's is nonzero.

Proof. $\theta_{1}$ and $\theta_{2}$ vanish only on $D_{1}$ and $D_{2}$ respectively so we may assume $R \in D_{1} \cap D_{2}$. If $\theta_{3}(R)=0$, we could find a splitting of

$$
0 \rightarrow M\left(-P_{1}-P_{2}\right)_{R} \rightarrow E_{R}^{\prime \prime} \rightarrow Q_{R} \rightarrow 0 .
$$

But such a splitting would give a splitting of (10.2.1) so that $M_{R}$ would coincide with $\left(N_{i}\right)_{R}$ over $P_{i}$. But $\left(N_{i}\right)_{R}=\left(L_{i}\right)_{R}$ and so we contradict our previous claim.

Let $\theta=\left(\theta_{1}, \theta_{2}, \theta_{3}\right)$ be the section of the bundle on $\tilde{Z}$ defined by

$$
G=\operatorname{Hom}\left(Q_{P_{2}}, M_{P_{1}}\right) \oplus \operatorname{Hom}\left(Q_{P_{1}}, M_{P_{2}}\right) \oplus R^{1} \pi_{*}\left(M \otimes Q^{-1}\left(-P_{1}-P_{2}\right)\right) .
$$

Now $Q=\tilde{\mathfrak{L}}_{\alpha} \otimes \mathfrak{R}^{\prime}$ and $M=\tilde{\mathfrak{L}}_{\alpha+1} \otimes \mathscr{I}_{\tilde{Z}} / \mathscr{G}_{\tilde{Z}}^{2}$, where $\Re^{\prime}$ is the pullback of the tautological bundle $\Re$ for $Z \rightarrow J_{\alpha+1} \times J_{\alpha}$. Consider the bundle $\mathscr{F}$ on $J_{\alpha} \times J_{\alpha+1}$ defined by

$$
\begin{aligned}
\mathscr{F}= & \operatorname{Hom}\left(\left(\varrho_{\alpha}\right)_{P_{1}},\left(\mathfrak{L}_{\alpha+1}\right)_{P_{2}}\right) \oplus \operatorname{Hom}\left(\left(\mathcal{L}_{\alpha}\right)_{P_{2}},\left(\mathcal{L}_{\alpha+1}\right)_{P_{1}}\right) \\
& \oplus R^{1} \pi_{*}\left(\mathcal{L}_{\alpha+1} \otimes \mathcal{L}_{\alpha}^{-1}\left(-P_{1}-P_{2}\right)\right) .
\end{aligned}
$$


Let $\mathscr{F}^{\prime}$ be the pullback of $\mathscr{F}$ to $Z$. Then

$$
G=p^{*}\left(\mathscr{F}^{\prime}\right) \otimes \mathscr{g}_{\tilde{Z}} / \mathscr{G}_{Z}^{2} \otimes \mathscr{T}^{-1}
$$

where $p$ is the projection from $\tilde{Z}$ to $Z$. Now using $\theta: \theta \rightarrow G$, we have a surjection

$$
p^{*}\left(\left(\mathscr{F}^{\prime}\right)^{-1} \otimes \Re\right) \rightarrow \mathscr{9}_{\tilde{Z}} / \mathscr{G}_{Z}^{2} \rightarrow 0
$$

and hence applying $p_{*}$, we see there is a surjective map

$$
\eta: \Re \otimes\left(\mathscr{F}^{\prime}\right)^{-1} \rightarrow p_{*}\left(\mathscr{g}_{\tilde{Z}} / \mathscr{G}_{Z}^{2}\right)=\mathscr{g}_{Z} / \mathscr{G}_{Z}^{2}
$$

on $Z$. Now by Riemann-Roch, the rank of $\mathscr{F}$ is $g+1$. On the other hand, $\operatorname{dim} S_{4}=4+\operatorname{dim} S_{0}=4 g-3$ and $\operatorname{dim} Z=2 g-4$ so $\mathrm{rk} \mathscr{F}=\operatorname{codim}_{S_{4}} Z$. Thus $\eta$ is an isomorphism since it is a surjective map between two bundles of the same rank. Since $\theta_{1}$ vanishes on $D_{1} \cap \tilde{Z}$, we see that $\operatorname{Hom}\left(\left(\mathcal{L}_{\alpha}\right)_{P},\left(\mathcal{L}_{\alpha+1}\right)\right)_{P_{2}}^{-1}$ $\otimes$ Tl corresponds to $\left(\mathscr{Q}_{D_{1}}\right)_{Z} \subseteq \mathscr{G}_{Z} / \mathscr{G}_{Z}^{2}$.

Thus $\tilde{Z}=\mathbf{P}\left(\mathscr{F}_{1}^{-1}\right) \times \mathbf{P}\left(\mathscr{F}^{-1}\right)$, where $\mathscr{F}_{1}$ is defined by (9.1.1). Let $p^{\prime}$ be the projection of $\tilde{Z}$ to $\mathbf{P}\left(\mathscr{F}^{-1}\right)$. Then Lemma 10.2 shows that $\psi$ is constant on the fibers of $p^{\prime}$, at least over $\tilde{Z}-D_{1}-D_{2}$. Now $D_{i}$ maps to a divisor in $\mathbf{P}\left(\mathcal{F}^{-1}\right)$, so $\psi$ is constant on all the fibers, since the pullback of any very ample bundle on $\vartheta_{P}$ is trivial on the fibers of $p^{\prime}$. One sees that $\psi$ restricted to $\tilde{Z}$ factors through $\mathbf{P}\left(\mathscr{F}^{-1}\right)$.

We claim $\psi$ maps $\mathrm{H}_{2}-\mathrm{D}_{2}$ onto the set of bundles $U\left(\mathrm{II}_{2}\right)$ of Type $\mathrm{II}_{2}$. Indeed, given a bundle $\tilde{F}_{R}$ on $X_{1}$ of Type $\mathrm{II}_{2}$, we can find a bundle $\tilde{F}$ on the surface $\mathcal{X}_{1}$ of $\S 5$ and an isomorphism $\varphi$ of $\tilde{F}_{H_{1}}$ with $\tilde{F}_{H_{2}}$ so that using $\varphi$ as glueing data produces $F_{R}$ over $R$. Now $\tilde{F}\left(+D_{2}\right)$ is the pullback of a family of stable bundles $F_{1}$ on $X \times S$ and we have

$$
\varphi:\left(F_{1}\right)_{P_{1}} \stackrel{\sim}{\rightarrow}\left(F_{1}\right)_{P_{2}}(-R) .
$$

The geometric realization of $\varphi$ over $R$ is just $F_{R}$. The data of $\varphi$ and $F$ define a map of $S$ to $S_{2}$ which passes transversally through $H_{2}-D^{(2)}$ at $R$. Since the map of $S_{4}$ to $S_{2}$ is a local isomorphism at any point of $H_{2}-D^{(2)}$, we get a map $\varphi^{\prime}$ of $S$ to $S_{4}$ so that $\psi \circ \varphi^{\prime}(R)$ is $F_{R}$. Similarly, $H_{1}-D_{1}$ maps onto bundles of Type $\mathrm{II}_{2}$.

We claim $H_{1} \cap D_{1}$ maps onto the set $U$ (III) of bundles of Type III. Indeed, $U(\mathrm{III}) \subseteq \overline{U\left(\mathrm{II}_{2}\right)}$, so $U\left(\right.$ III) is in the (closed) image of $H_{1}$. But no element of $H_{1}-D_{1}$ maps to $U(\mathrm{III})$, so $H_{1} \cap D_{1}$ maps onto $U(\mathrm{III})$. Next notice that $D_{1}$ maps onto $\overline{U\left(\mathrm{II}_{1}\right)}$. Indeed, $\psi\left(D_{1}\right)$ is strictly larger than $U(\mathrm{III})$ since no element of $D_{1}-H_{1}-H_{2}$ maps to $U(\mathrm{III})$. Since $U(\mathrm{III})$ is a divisor in $\overline{U\left(\mathrm{II}_{1}\right)}$, we see $D_{1}$ maps onto $\overline{U\left(\mathrm{II}_{1}\right)}$. Similarly, $D_{1}$ maps onto $\overline{U\left(\mathrm{II}_{1}\right)}$. 
Lemma 10.5. If $D$ is a divisor on $S_{4}, D \neq \tilde{Z}$, then $\psi(D)$ is a divisor. Further, $\psi$ restricted to $\tilde{Z}$ factors through $\mathbf{P}\left(\mathscr{F}^{-1}\right)$.

11. Suppose that $\tilde{S}$ and $S^{\prime}$ are smooth projective varieties and that $\varphi$ : $\tilde{S} \rightarrow S^{\prime}$ is a birational morphism. Suppose $Z$ is smooth variety and that $\mathscr{F}$ is a bundle on $Z$. Suppose $\tilde{Z}$ is a division on $\tilde{S}$ and that we can identify $\tilde{Z}$ with $\mathbf{P}\left(\mathscr{F}^{-1}\right)$ in such a way that $\oint_{\tilde{Z}} / \mathscr{G}_{Z}^{2}$ is the tautological bundle on $\mathbf{P}\left(\mathscr{F}^{-1}\right)$. [In our application, $\tilde{S}=S_{4}, S^{\prime}=\tilde{w_{P}}$, the normalization of $\mathcal{w}_{P}$.]

Lemma 11.1. Suppose that if $D \subseteq \tilde{S}$ is any divisor with $D \neq \tilde{Z}$, then $\varphi(D)$ is a divisor. Suppose further that the induced map of $\tilde{Z}$ to $S^{\prime}$ factors through $Z$. Then the map of $Z$ to $S^{\prime}$ is an inclusion, and $\tilde{S}$ is the blow up of $S^{\prime}$ at $Z$.

Proof. Let $\psi: \varphi^{*}\left(\Omega_{S^{\prime}}\right) \rightarrow \Omega_{\tilde{S}}$ be the induced map. Let $U$ be the set of points over which $\psi$ is an isomorphism. Then $D=\tilde{S}-U$ is a divisor given by $\operatorname{det} \psi=0$. By Zariski's main theorem, we see that $\varphi^{-1}(\varphi(U))=U$ and that for each $P \in D, \varphi^{-1}(\varphi(P))$ is connected and has dimension $\geqslant 1$. Thus $D=\tilde{Z}$. Let $\pi$ be projection of $\tilde{Z}$ to $Z$.

Now consider $\Omega_{0}=\operatorname{Ker}\left(\Omega_{\tilde{S}}, \Omega_{\tilde{Z} / Z}\right)$. The image of $\psi$ is contained in $\Omega_{0}$ since locally any function on $S^{\prime}$ is constant on the fibers of $\pi$. Now $\theta(1)=\Phi_{\tilde{Z}} / g_{Z}^{2}$. We first claim that det $\Omega_{0}$ is trivial on the fibers of $\pi$. Indeed, we have the following exact sequences:

$$
\begin{gathered}
0 \rightarrow \vartheta(1) \rightarrow\left(\Omega_{\tilde{S}}\right)_{\tilde{Z}} \rightarrow \Omega_{\tilde{Z}} \rightarrow 0, \\
0 \rightarrow \pi^{*} \Omega_{Z} \rightarrow \Omega_{\tilde{Z}} \rightarrow \Omega_{\tilde{Z} / Z} \rightarrow 0, \\
0 \rightarrow \Omega_{\tilde{Z} / Z}(+1) \rightarrow \pi^{* \mathscr{F}-1} \rightarrow \vartheta(1) \rightarrow 0, \\
0 \rightarrow \Omega_{\tilde{Z} / Z}(+1) \rightarrow\left(\Omega_{0}\right)_{\tilde{Z}} \rightarrow\left(\Omega_{\tilde{S}}\right)_{\tilde{Z}} \rightarrow \Omega_{\tilde{Z} / Z} \rightarrow 0 .
\end{gathered}
$$

So in $K(\tilde{Z})$, we have

$$
\left[\left(\Omega_{0}\right)_{\tilde{Z}}\right]=\left[\pi^{*} \Omega_{Z}\right]+\left[\pi^{*} \mathscr{F}^{-1}\right] .
$$

Hence det $\Omega_{0}$ is trivial on the fibers of $\pi$. Now the map from det $\varphi^{*} \Omega_{S^{\prime}}$ to $\operatorname{det} \Omega_{0}$ is an isomorphism outside $\tilde{Z}$, so

$$
\operatorname{det}\left(\varphi^{*} \Omega_{S^{\prime}}\right)=\left(\operatorname{det} \Omega_{0}\right)(-n \tilde{Z}) .
$$

But $\theta(-n \tilde{Z})$ is nontrivial on the fibers of $\pi$ if $n \neq 0$. So $n=0$ and $\operatorname{det} \varphi^{*}\left(\Omega_{S^{\prime}}\right)$ $=\operatorname{det} \Omega_{0}$. Thus $\varphi^{*}\left(\Omega_{S^{\prime}}\right)=\Omega_{0}$.

The map $\varphi_{Z}$ from $Z$ to $S^{\prime}$ has connected fibers since the map from $\tilde{Z}$ to $S^{\prime}$ has connected fibers. On the other hand, the map from $\varphi_{Z}^{*}\left(\Omega_{S^{\prime}}\right)$ to $\Omega_{Z}$ is surjective, since $\pi^{*} \Omega_{Z}$ is a quotient of $\Omega_{0}$ and hence of $\varphi^{*} \Omega_{S}$. Thus $\varphi_{Z}$ is an embedding. We identify $Z$ with its image. There is an exact sequence on $S^{\prime}$

$$
0 \rightarrow g_{Z} / g_{Z}^{2} \rightarrow\left(\Omega_{S^{\prime}}\right)_{Z} \rightarrow \Omega_{Z} \rightarrow 0
$$


Now $\operatorname{Ker}\left(\left(\Omega_{0}\right)_{\tilde{Z}}, \varphi^{*} \Omega_{Z}\right)$ surjects to $\Phi_{\tilde{Z}} / g_{Z}^{2}$, and hence $\varphi^{*}\left(\dot{g}_{Z} / g_{Z}^{2}\right)$ maps surjectively to $\Phi_{\tilde{Z}} / \Phi_{\tilde{Z}}^{2}$. Thus, $\varphi^{*}\left(\Phi_{Z}\right)=g_{\tilde{Z}}$. Let $\tilde{S}^{\prime}$ be the blow up of $S$ at $Z$ and let $E$ be the exceptional divisor. By the universal property of blowing up, $S$ maps to $\tilde{S}^{\prime}$ and $\tilde{Z}$ maps onto $E$ and is therefore generically finite. As before, the map from the pullback of $\Omega_{\tilde{S^{\prime}}}$ is an isomorphism, and hence $\tilde{S}=\tilde{S}^{\prime}$.

12. Let $S$ be a smooth projective variety and suppose $D_{1}, \cdots, D_{n}$ are smooth divisors which intersect transversally. Suppose $Z \subseteq D_{1} \cap D_{2}$ is a smooth subvariety and $Z \cap D_{k}=\varnothing$ for $k>2$. Further, suppose $E$ and $F$ are bundles on a smooth variety $J$ and let $Z=\mathbf{P}(E)$. Let $\pi: Z \rightarrow J$ be the projection and let $\theta(1)$ be the tautological bundle for $\pi$. Suppose $g_{Z} / g_{Z}^{2}=$ $\pi^{*}(F)(+1)$ and there are line bundles $L_{i} \subseteq F$ so that $\Phi_{D_{i}} \otimes \theta_{Z}$ corresponds to $\pi^{*}\left(L_{i}\right)(+1)$.

Blow up $Z$ on $S$ to obtain $\tilde{S}$ with new exceptional divisor $\tilde{Z}$. Let $\tilde{D}_{i}$ be the proper transforms of $D_{i}$. Note that $\tilde{Z}=\mathbf{P}(E) \times{ }_{J} \mathbf{P}(F)$. We will assume there is a map $\varphi$ from $\tilde{S}$ to another smooth variety so that $\varphi_{\tilde{Z}}$ factors through $\mathbf{P}(F)=Z^{\prime}$ and so that $\varphi(D)$ is a divisor on $S^{\prime}$ if $D \neq \tilde{Z}$. From $\S 11$, we see that $Z^{\prime} \subseteq S^{\prime}$ and that $\tilde{S}$ is the blow up of $S^{\prime}$ at $Z^{\prime}$. Let $D_{i}^{\prime}$ be the images of $\tilde{D}_{i}$ in $S^{\prime}$. First, notice that the $D_{i}^{\prime}$ intersect transversally. Indeed, $D_{1}^{\prime} \cap Z^{\prime}$ and $D_{2}^{\prime} \cap Z^{\prime}$ are the divisors on $Z^{\prime}=\mathbf{P}(F)$ corresponding to the subbundles $L_{1}$ and $L_{2}$ of $F$.

Now let $k=\operatorname{dim} J$, let $m=\operatorname{dim} S$ and let $D=\sum D_{i}$. Our main goal in this section is

Proposition 12.1. If $c_{i}\left(\Omega_{S}(\log D)\right)=0$ for $i \geqslant m-k-1$ and $c_{i}\left(\Omega_{J}\right)=0$ for $i>0$, then $c_{i}\left(\Omega_{S^{\prime}}\left(\log D^{\prime}\right)\right)=0$ for $i \geqslant m-k-1$.

The Chern classes may be taken in any convenient cohomology theory.

We begin with the following well-known consequence of Grothendieck's Riemann-Roch Theorem. Let $X$ be a smooth projective variety and let $H$ be a smooth divisor on $X$. Let $E$ be a bundle of rank $r$ on $H$ and let $c(E)=$ $\Pi\left(1+a_{i}\right)$ be the Chern polynomial of $E$ on $H$, where the product is the usual formal device. Let $h$ be the divisor class of $\mathcal{O}_{H}(H)$ on $H$ and let $i$ be the inclusion of $H$ into $X$.

Lemma 12.2.

$$
c\left(i_{*} E\right)=1+i_{*}\left(\frac{1-\Pi\left(\left(1+a_{i}\right) /\left(1+a_{i}-h\right)\right)}{h}\right) .
$$

Proof. Let $\eta_{X}^{-1}$ be the operator on $A(X)$ which multiplies a class of degree $i$ by $(-1)^{i-1} \cdot(i-1) !$. Then

$$
c\left(i_{*} E\right)=\exp \left(\eta_{X}^{-1} \cdot \operatorname{ch}\left(i_{*} E\right)\right) .
$$


From Grothendieck's Riemann-Roch formula for $i$, we know

$$
\operatorname{ch}\left(i_{*} E\right)=i_{*}\left(\operatorname{ch} E \cdot \frac{1-e^{-h}}{h}\right) \text {. }
$$

Now in general

$$
e^{i_{*} \alpha}=1+i_{*}\left(\left(1-e^{\alpha \cdot h}\right) / h\right)
$$

Hence

$$
\begin{aligned}
c\left(i_{*} E\right) & =\exp \left(\eta_{X}^{-1}\left(i_{*}\left(\operatorname{ch} E \cdot \frac{1-e^{-h}}{h}\right)\right)\right)=\exp i_{*}\left(\frac{\eta_{H}^{-1}\left(\operatorname{ch} E \cdot\left(1-e^{-h}\right)\right)}{h}\right) \\
& =1+i_{*}\left(\frac{1+\Pi\left(\left(1+a_{i}\right) /\left(1+a_{i}-h\right)\right)}{h}\right)
\end{aligned}
$$

since $\operatorname{ch} E=\sum e^{a_{i}}$.

Suppose $F$ is a bundle on $X$ and that we have an exact sequence

$$
0 \rightarrow F_{1} \rightarrow F_{H} \rightarrow F_{0} \rightarrow 0
$$

of bundles on $H$. Let $F^{\prime}=\operatorname{Ker}\left(F, F_{0}\right)$. Let $r_{1}$ be the rank of $F_{1}$ and $r$ be the rank of $F_{0}$.

Lemma 12.3. Suppose $c_{i}\left(F_{1}\right)=0$ as a Chern class on $H$ for $i \geqslant r_{1}-k$, where $k$ is some positive integer. Then $c_{i}(F)=c_{i}\left(F^{\prime}\right)$ on $X$ for $i \geqslant r_{1}+r-k$.

Proof. On $H$, we write formally $c\left(F_{0}\right)=\Pi^{r}\left(1+a_{i}\right)$. Then

$$
c\left(F_{0}^{\prime}\right)=c\left(F_{1}\right) \cdot \Pi\left(1+a_{i}-h\right),
$$

since there is an exact sequence

$$
0 \rightarrow F_{0}(-H) \rightarrow F_{H}^{\prime} \rightarrow F_{1} \rightarrow 0 .
$$

Now $c(F)=c\left(F^{\prime}\right) \cdot c\left(i_{*} F_{0}\right)$. Thus

$$
\begin{aligned}
c(F)-c\left(F^{\prime}\right) & =c\left(F^{\prime}\right) \cdot\left(c\left(i_{*}\left(F_{0}\right)\right)-1\right) \\
& =c\left(F^{\prime}\right) i_{*}\left(\frac{1-\Pi\left(1+a_{i}\right) /\left(1+a_{i}-h\right)}{h}\right) \\
& =i_{*}\left(\frac{1}{h}\left\{\left(\prod^{r}\left(1+a_{i}-h\right)-\prod^{r}\left(1+a_{i}\right)\right) c\left(F_{1}\right)\right\}\right) .
\end{aligned}
$$

We see the expression in the braces has no components in degrees greater than $r+r_{1}-k$.

Next we remark that if $f$ is a rational function on $P^{k}$, and if the divisor of $f$ is $H_{1}-H_{0}$ where $H_{1}$ and $H_{0}$ are hyperplanes, then $d \log f$ is a nowhere zero section of $\Omega\left(\log \left(H_{0}+H_{1}\right)\right)$. Indeed, if $\left\{X_{i}\right\}$ are homogeneous coordinates with 
$H_{i}=\left\{X_{i}=0\right\}$ for $i=0,1$, then if $P \notin H_{1} \cup H_{2}$, then $f$ is a coordinate function and so $d f \neq 0$ at $P$. On the other hand, if $P \in H_{1}$, then the residue of $f$ around $H_{1}$ is nonzero.

We will next construct a canonical section $s$ of $\Omega_{\tilde{Z} / J}\left(\log \left(\tilde{D}_{1}^{\prime}+\tilde{D}_{2}^{\prime}\right)\right)$, where $\tilde{D}_{i}^{\prime}=\tilde{D_{i}} \cap \tilde{Z}$. Now consider $z \in Z$ and let $w$ be the projection of $z$ in $J$. Now $\left(\Phi_{D_{1}} \otimes g_{D_{2}}^{-1}\right)_{Z}=\pi^{*}\left(L_{1} \otimes L_{2}^{-1}\right)$. Let $\left(f_{1}, f_{2}\right)$ be a pair of functions defined in a neighborhood of $z$ with $f_{i}$ vanishing simply on $D_{i}$. We say $\left(f_{1}, f_{2}\right)$ is good if $f_{1} \otimes f_{2}^{-1}$ as a section of $\left(g_{D_{1}} \otimes g_{D_{2}}^{-1}\right)_{Z}$ is the pullback of a section of $L_{1} \otimes L_{2}^{-1}$. Note that if $f_{1}^{\prime}$ and $f_{2}^{\prime}$ are another good pair, then

$$
\frac{z_{1}}{z_{2}}=\frac{z_{1}^{\prime}}{z_{2}^{\prime}}+f
$$

where $f$ is a unit and $f$ restricted to $Z$ is the pullback of a function on $J$. Thus $d \log \left(z_{1} / z_{2}\right)$ gives a section $s$ of $\Omega_{\tilde{Z} / J}\left(\log \left(\tilde{D}_{1}^{\prime}+\tilde{D}_{2}^{\prime}\right)\right)$ which is independent of the good pair. The above remark shows that $s$ projects to a nowhere zero section of $\Omega_{\tilde{Z} / Z}\left(\log \left(\tilde{D}_{1}^{\prime}+\tilde{D}_{2}^{\prime}\right)\right)$. On the other hand, let $\bar{s}$ denote the projection of $s$ in $\Omega_{\tilde{Z} / Z^{\prime}}\left(\log \left(\tilde{D}_{1}^{\prime}+\tilde{D}_{2}^{\prime}\right)\right)$. We claim $\bar{s}=0$. Indeed, $\Omega_{\tilde{Z} / Z^{\prime}}$ is a negative bundle on the fibers of $\pi^{\prime}: \tilde{Z} \rightarrow Z^{\prime}$, so $\bar{s}$ vanishes on $\tilde{Z}-\left(\pi^{\prime}\right)^{-1}\left(D_{1}^{\prime}+D_{2}^{\prime}\right)$. Hence $\bar{s}$ vanishes. Now let $F \subseteq \Omega_{\tilde{Z}}\left(\log \left(\tilde{D}_{1}+\tilde{D}_{2}\right)\right)$ be the subsheaf generated locally by $\Omega_{J}$ and $d \log \left(z_{1} / z_{2}\right)$. Then we have an exact sequence

$$
0 \rightarrow \pi^{*} \Omega_{J} \rightarrow F \rightarrow \theta_{\tilde{Z}} \rightarrow 0 .
$$

Also, let $L \subseteq \Omega_{\tilde{Z} / Z}\left(\log \left(\tilde{D}_{1}^{\prime}+\tilde{D}_{2}^{\prime}\right)\right)$ be the subline bundle generated by $s$, so $L \cong \theta_{\tilde{Z}}$.

Consider on $Q$ the sheaf defined by

$$
0 \rightarrow \pi^{*} \Omega_{S}(\log D) \rightarrow \Omega_{\tilde{S}}(\log (\tilde{D}+\tilde{Z})) \rightarrow Q \rightarrow 0 .
$$

Lemma 12.4. $Q \cong \Omega_{\tilde{Z} / Z}\left(\log \left(\tilde{D}_{1}^{\prime}+\tilde{D}_{2}^{\prime}\right)\right) / L$.

Proof. Consider the following diagram:

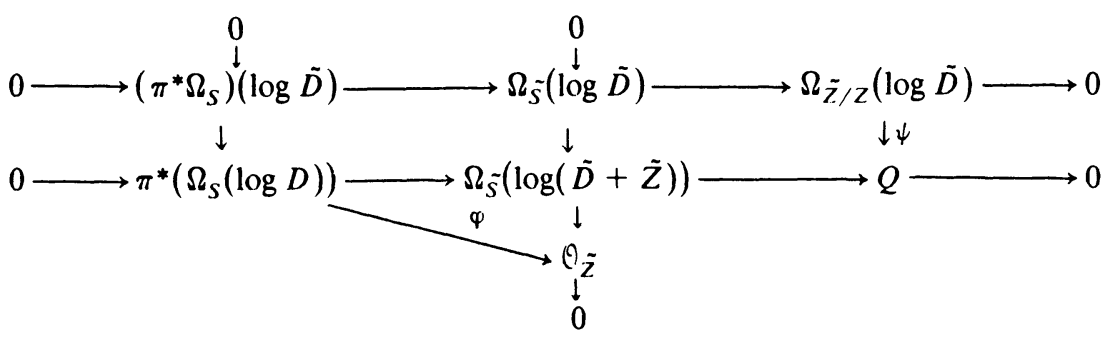

We will show that $\psi(L)=0$ and the induced map $\bar{\psi}$ of $\Omega_{\tilde{Z} / Z}(\log \tilde{D}) / L$ to $Q$ is the required isomorphism. We first claim that $\varphi$ is onto. Let $\tilde{z} \in \tilde{Z}$ and let $z$ be projection of $z$ in $Z$. Let $\left(f_{1}, f_{2}\right)$ be a good pair defined in a neighborhood of $z$. If $\tilde{z} \notin \tilde{D}_{1}$, then $f_{1}$ is a local equation for $\tilde{Z}$, so $d \log f_{1}$ generates $\theta_{\tilde{Z}}$ at these 
points. If $\tilde{z} \in \tilde{D}_{1}$, then $f_{1}=t t^{\prime}$ where $t$ and $t^{\prime}$ are local equations for $\tilde{D}_{1}$ and $\tilde{Z}$. Thus $d \log t$ is a section of $\Omega(\log \tilde{D})$, so since $d \log t^{\prime}=d \log f_{1}-d \log t$, we see that $\varphi$ is onto. We further claim that any local section $s$ of $\Omega_{\tilde{S}}(\log (\tilde{D}))$ which is also a section of $\pi^{*}\left(\Omega_{S}(\log D)\right)$ maps to $L \subseteq \Omega_{\tilde{Z} / Z}(\log \tilde{D})$. Indeed, note that if $\varphi\left(h_{1} d\left(\log f_{1}\right)-h_{2} d\left(\log f_{2}\right)\right)=0$, then we must have $h_{1}=h_{2}$ on $\tilde{Z}$. Since all the sections of $\pi^{*} \Omega_{S}$ map to zero in $\Omega_{\tilde{Z} / Z}(\log \tilde{D})$, our claim follows. Finally, note that $\psi(L)=0$ since $d \log \left(f_{1} / f_{2}\right) \in \pi^{*}\left(\Omega_{S}(\log D)\right)$. It follows that $\bar{\psi}$ is an isomorphism.

Proof of Proposition 12.1. First note that

$$
0 \rightarrow \Phi_{\tilde{Z}} / \mathscr{q}_{\tilde{Z}}^{2} \rightarrow\left[\Omega_{\tilde{S}}(\log \tilde{D})\right]_{\tilde{Z}} \stackrel{\lambda}{\rightarrow}\left[\Omega_{\tilde{S}}(\log (\tilde{D}+\tilde{Z}))\right]_{\tilde{Z}} \rightarrow \theta \rightarrow 0
$$

is exact and that the image of $\lambda$ is $\Omega_{\tilde{z}}\left(\log \left(\tilde{D}_{1}+\tilde{D}_{2}\right)\right)$. Thus, $F$ is a subbundle of $\Omega_{\tilde{s}}(\log (\tilde{D}+\tilde{Z}))_{\tilde{Z}}$. Further, $F$ maps to zero in $Q$. Applying Lemma 12.3, and using the fact that $c_{j}(F)=0$ for $j>0$, we see that

$$
0=\pi^{*}\left(c_{i}\left(\Omega_{S}(\log D)\right)\right)=c_{i}\left(\pi^{*} \Omega_{S}(\log D)\right)=c_{i}\left(\Omega_{\tilde{S}}(\log (\tilde{D}+\tilde{Z}))\right)
$$

for $i \geqslant m-k-1$, since rk $F=k+1$. Now $F$ maps to zero in the quotient $\theta_{\tilde{Z}}$ of $\Omega_{\tilde{S}}(\log (\tilde{D}+\tilde{Z}))$ since $d \log f_{1} / f_{2}$ does not have a pole on $\tilde{Z}$. Using a similar argument, we have

$$
0=c_{i}\left(\Omega_{\tilde{S}}(\tilde{D}+\tilde{Z})\right)=c_{i}\left(\Omega_{\tilde{S}}(\tilde{D})\right)
$$

for $i \geqslant m-k-1$. Now we also have an exact sequence

$$
0 \rightarrow \pi^{\prime *}\left(\Omega_{S^{\prime}}\left(\log D^{\prime}\right)\right) \rightarrow \Omega_{S}(\log \tilde{D}) \rightarrow \Omega_{\tilde{Z} / Z^{\prime}}(\log (\tilde{D})) \rightarrow 0 .
$$

Now $F \subseteq\left[\Omega_{S}(\log \tilde{D})\right]_{\tilde{Z}}$ maps to zero in $\Omega_{\tilde{Z} / Z^{\prime}}(\log \tilde{D})$ by the above remark that $\bar{s}=0$. Now applying Lemma 12.3 again, we see that

$$
0=c_{i}\left(\Omega_{S}(\log \tilde{D})\right)=c_{i}\left(\pi^{*}\left(\Omega_{S^{\prime}}\left(\log D^{\prime}\right)\right)\right)=c_{i}\left(\Omega_{S^{\prime}}\left(\log D^{\prime}\right)\right)
$$

for $i \geqslant m-k-1$.

13. Continuing with the notation of $\S 10$, let $Z^{\prime}=\mathbf{P}\left(\mathscr{F}^{-1}\right)$ and let $\tilde{\tilde{f}_{P}}$ be the normalization of $\mho_{P}$. The map $\psi$ from $S_{4}$ to $\mho_{P}$ factors $\tilde{W}_{P}$, since $S_{4}$ is normal. Let $\tilde{\psi}$ be the induced map of $S_{4}$ to $\tilde{\psi}_{P}$.

Theorem 13.1. $Z^{\prime}$ is a subvariety of $\tilde{\mathcal{O}} S_{P}$, and $S_{4}$ is the blow up of $\tilde{\mathscr{O}} \int_{P}$ at $Z^{\prime}$. Further, if $D^{\prime} \subseteq$ ข̃ $\int_{P}$ is the divisor which maps to the singular locus of $\mathscr{W}_{P}$, then $H_{1}+H_{2}+D_{1}+D_{2}$ is the inverse image of $D^{\prime}$.

Corollary 13.2. If $c_{i}\left(\Omega_{S_{3}}\left(\log \left(H_{1}+H_{2}+D_{1}+D_{2}\right)\right)\right)=0$ for $i>2 g-2$, then $c_{i}\left(\Omega_{\tilde{\vartheta}_{p}}\left(\log D^{\prime}\right)\right)=0$ for $i>2 g-2$.

Theorem 13.1 follows from $\$ 11$ and Corollary 13.2 from $\S 12$.

Proposition 13.3. $\quad c_{i}\left(\Omega_{\tilde{\omega}_{P}}\left(\log \left(D^{\prime}\right)\right)\right)=0$ if $i>2 g-2$ if $c_{i}\left(\Omega_{S_{0}}\right)=0$ for $i>$ $2(g-1)-2$. 
Proof. We will show first that

$$
c_{3}\left(\Omega_{S_{1} / S_{0}}(\log D)\right)=0 .
$$

We will work in the Chow ring modulo algebraic equivalence. First, notice that $E_{P_{1}}$ and $E_{P_{2}}$ are algebraically equivalent by letting $P$ move from $P_{1}$ to $P_{2}$. Hence, we may formally write

$$
c\left(E_{P_{i}}\right)=\left(1+a_{1}\right)\left(1+a_{2}\right) .
$$

Let $\alpha=a_{1}-a_{2}$ and let $\xi$ be the divisor class of $M_{1}$ on $S_{1}$. Thus $D=2 \xi$, since $\wedge{ }^{2} E_{P_{1}}$ and $\wedge \wedge^{2} E_{P_{2}}$ are algebraically equivalent. Let $F=E_{P_{2}}^{-1} \otimes E_{P_{1}}$. Then we have the following exact sequences on $S_{1}$ :

$$
\begin{gathered}
0 \rightarrow \Omega_{S_{1} / S} \rightarrow \pi_{1}^{*}(F)(-\xi) \rightarrow \theta \rightarrow 0, \\
0 \rightarrow \Omega_{S_{1} / S} \rightarrow \Omega_{S_{1} / S}(\log D) \rightarrow \vartheta_{D} \rightarrow 0, \\
0 \rightarrow \vartheta(-D) \rightarrow \theta \rightarrow \vartheta_{D} \rightarrow 0 .
\end{gathered}
$$

Further, $\xi^{2}\left(\xi^{2}-\alpha^{2}\right)=0$ so

$$
c(F(-\xi))=(1-\xi)^{2}\left((1-\xi)^{2}-\alpha^{2}\right) .
$$

Thus

$$
\begin{aligned}
& c\left(\Omega_{S_{1} / S}(\log D)\right)=\frac{(1-\xi)^{2}\left((1-\xi)^{2}-\alpha^{2}\right)}{1-2 \xi} \\
& \quad=\frac{1}{1-2 \xi}\left[(1-2 \xi)\left((1-\xi)^{2}-\alpha^{2}\right)+\xi^{2}(1-2 \xi)+\xi^{2}\left(\xi^{2}-\alpha^{2}\right)\right] \\
& \quad=\left((1-\xi)^{2}-\alpha^{2}\right)+\xi^{2} .
\end{aligned}
$$

Thus $c_{3}\left(\Omega_{S_{1} / S_{0}}(\log D)\right)=0$.

We next note that $\Omega_{S_{2} / S_{1}}\left(\log \left(H_{1}+H_{2}\right)\right) \cong \theta$. Indeed, we can find a cover $U_{i}$ of $S_{1}$ so that there are rational functions $z_{i}$ on $\pi_{2}^{-1}\left(U_{i}\right)$ with a simple pole on $H_{1}$ and a simple zero on $H_{2}$. On $\pi_{2}^{-1}\left(U_{i} \cap U_{j}\right)$,

$$
z_{i}=z_{j} \cdot f_{i j},
$$

where the $f_{i j}$ are units on $U_{i} \cap U_{j}$. Hence $d z_{i}$ give a well-defined and nowhere zero section of $\Omega_{S_{2} / S_{1}}\left(\log \left(H_{1}+H_{2}\right)\right.$ ).

Now $\Omega_{\mathrm{S}_{2}}\left(\log \left(D^{(2)}+H_{1}+H_{2}\right)\right)$ has a filtration whose successive quotients are $\Omega_{\mathrm{S}_{2} / \mathrm{S}_{1}}\left(\log \left(H_{1}+H_{2}\right)\right), \pi_{2}^{*}\left(\Omega_{\mathrm{S}_{1} / \mathrm{S}_{0}}(\log D)\right)$ and $\pi_{2}^{*} \pi_{1}^{*}\left(\Omega_{S_{0}}\right)$. Hence

$$
c_{i}\left(\Omega_{S_{2}}\left(\log \left(D^{(2)}+H_{1}+H_{2}\right)\right)\right)=0
$$

if $i>2 g-2$. 
Now letting $E$ be the divisor $D_{1}+D_{2}+H_{1}+H_{2}$ on $S_{3}$, a local computation shows that

$$
\pi^{*} \Omega_{S_{2}}\left(\log \left(D^{(2)}+H_{1}+H_{2}\right)\right)=\Omega_{S_{3}}(\log E) .
$$

Applying Corollary 13.2 establishes Proposition 13.3.

Proof of Theorem 1.1. From Atyiah's classification of stable bundles on elliptic curves, the moduli space $U_{X}$ is just $X$ if $X$ is an elliptic curve. Thus, Theorem 1.1 is true for $g=1$.

Assume that the theorem is true for genus $g-1$. There is a vector bundle $\Omega_{\text {W/C }}\left(\log D^{\prime}\right)$ so that for $R \in C, R \neq P,\left(\Omega_{\mho / C}\left(\log D^{\prime}\right)\right)_{R}$ is just the sheaf of one forms on the moduli space of stable vector bundles of degree $2 \alpha+1$ on $\mathcal{X}_{R}$. Now $\mathcal{W}_{P}$ is a deformation retract of $\mathscr{W}$, so to show $c_{i}\left(\Omega_{\mho / C}\left(\log D^{\prime}\right)\right)=0$, it suffices to show $c_{i}\left(\Omega_{\mathscr{W}_{P}}\left(\log D^{\prime}\right)\right)=0$. Once this is done for $i>2 g-2$, Theorem 1.1 will be established for $\mathfrak{X}_{R}$. But once Theorem 1.1 is established for one smooth curve, it is established for any smooth curve.

Thus Theorem 1.1 will follow from

Lemma 13.4. Let $X$ be a projective scheme over $\mathrm{C}$ with normal crossings and let $E$ be the bundle $X$. Suppose the $X_{j}$ are the normalizations of the irreducible components of $X$. Then if $E_{j}$ is the pullback of $E$ to $X_{j}$, and $c_{i}\left(E_{j}\right)=0$ for all $j$, then $c_{i}(E)=0$.

Proof. Let $2 i=n$. Recall that there is functorial mixed Hodge structure on $H^{n}(X, \mathrm{C})$. Thus, there is a weight filtration

$$
\cdots \subseteq W_{n-1} \subseteq W_{n}=H^{n}(X, \mathbf{Q})
$$

and a Hodge filtration

$$
0 \subseteq F^{n} \subseteq F^{n-1} \subseteq \cdots \subseteq F^{0}=H^{n}(X, \mathbf{C})
$$

Now the natural map of $H^{n}(X, \mathbf{Q})$ to $H^{n}(\tilde{X}, \mathbf{Q})$ induces an injective map from $W_{n} / W_{n-1}$ to $H^{n}(\tilde{X}, \mathbf{Q})$. Thus $c_{i}(E) \in W_{n-1}$, since $c_{i}(E)$ goes to zero in $H^{n}(\tilde{X}, \mathbf{Q})$.

On the other hand, we claim $c_{i}(E) \in F^{i}$. Indeed, let $L$ be a very ample line bundle on $X$. Then we can find a map of $X$ to a grassmannian $G$ so that $E \otimes L^{n}$ is the pullback of the universal bundle for some $n$. We can find a map of $X$ into $\mathbf{P}^{N}$ so that $L^{n}$ is the pullback of $\theta(1)$. Now let $\mathcal{E}$ be the universal bundle on $G$, and consider $\varepsilon(-1)$ on $G \times \mathbf{P}^{N}$. Then we can map $X$ to $G \times \mathbf{P}^{N}$ so that $E$ is the pullback of $\mathscr{E}(-1)$. Since $c_{i}(\mathcal{E}(-1))$ is in the $i$ th level of the Hodge filtration and since the map of $H^{n}\left(G \times \mathbf{P}^{n}, \mathbf{Q}\right)$ to $H^{n}(X, \mathbf{Q})$ is a morphism of Hodge structures, $c_{i}(E) \in F^{i}$.

Let $F_{n-1}^{k}$ be the induced Hodge filtration on $W_{n-1} / W_{n-2}$. Then $F_{n-1}^{k}$ is a pure Hodge structure of weight $n-1$. In particular, $\cdot F_{n-1}^{i} \cap F_{n-1}^{i}=0$. Thus $c_{i}(E) \in W_{n-2}$. Continuing this line of reasoning, we see that $c_{i}(E)=0$. 


\section{References}

[1] D. Gieseker \& I. Morrison, Hilbert stability of rank two bundles on curves, J. Differential Geometry 19 (1984).

[2] D. Mumford \& J. Fogarty, Geometric invariant theory, 2nd ed., Springer, Berlin, 1982.

[3] P. E. Newstead, Introduction to moduli problems and orbit spaces, Tata Lecture Notes, Narosa, 1978.

University of CALIFornia, Los ANGeles 